UT-855

RESCEU-28/99

\title{
Leptogenesis in Inflationary Universe
}

\author{
T. Asaka, ${ }^{1}$ K. Hamaguchi, ${ }^{1}$ M. Kawasaki ${ }^{2}$ and T. Yanagida ${ }^{1,2}$ \\ ${ }^{1}$ Department of Physics, University of Tokyo, Tokyo 113-0033, Japan \\ ${ }^{2}$ Research Center for the Early Universe, University of Tokyo, Tokyo 113-0033, Japan
}

(July 30, 1999)

\begin{abstract}
We investigate the leptogenesis via decays of heavy Majorana neutrinos which are produced non-thermally in inflaton decays. We make a comprehensive study on the leptogenesis assuming various supersymmetric (SUSY) models for hybrid, new and topological inflations. For an estimation of the lepton asymmetry we adopt the Froggatt-Nielsen mechanism for mass matrices of quarks and leptons. We find that all of these models are successful to produce the lepton asymmetry enough to explain the baryon number in the present universe. Here we impose low reheating temperatures such as $T_{R} \lesssim 10^{8} \mathrm{GeV}$ in order to suppress the abundance of gravitinos not to conflict with the bigbang nucleosynthesis. Furthermore, we find that the leptogenesis works very well even with $T_{R} \simeq 10^{6} \mathrm{GeV}$ in the SUSY hybrid or new inflation model. It is known that such a reheating temperature is low enough to suppress the abundance of gravitinos of mass $m_{3 / 2} \simeq 100 \mathrm{GeV}-1 \mathrm{TeV}$. Thus, the leptogenesis is fully consistent with the big-bang nucleosynthesis in a wide region of the gravitino mass.
\end{abstract}




\section{INTRODUCTION}

It was first pointed out by 'tHooft in 1976 that instanton-like nonperturbative effects violate baryon-number conservation in the standard electroweak gauge theory [1]. These effects are exponentially suppressed by a large factor at zero temperature and hence the stability of proton is practically guaranteed. Kuzmin, Rubakov and Shaposhnikov suggested [2], however, that the baryon-number violation is not suppressed and can be even efficient at high temperatures above the electroweak phase transition. These baryon-number violating ("sphaleron") processes conserve a linear combination of baryon $(B)$ and lepton $(L)$ numbers, that is, $B-L$. Therefore, if the baryon asymmetry was generated in the early universe with $B-L$ conserving processes as in the standard $S U(5)$ grand unified theory, all baryon asymmetry would be washed out by the "sphaleron" effects [1. The existence of baryon (matter-antimatter) asymmetry in the present universe, thus, indicates that the $B-L$ violating processes played an important role on generating the baryon asymmetry in the early universe.

It is quite natural to consider that such $B-L$ violating interactions induce effective operators of $B-L$ violation at low energies. The lowest dimensional possible operators in the electroweak gauge theory is

$$
\mathcal{O}=\frac{f_{i j}}{M} l_{i} l_{j} H H
$$

where $l_{i}(i=1,2,3)$ and $H$ denote lepton doublets and the Higgs scalar field, respectively, and $M$ is the scale of $B-L$ violation. These operators induce small neutrino masses in the vacuum $\langle H\rangle \simeq 246 / \sqrt{2} \mathrm{GeV}$ [6]. There is now a convincing experimental evidence [7] that one of neutrinos has indeed a small mass $m_{\nu}$ of order $0.1 \mathrm{eV}$, which would encourage us to continue the above consideration to some extent.

\footnotetext{
1 This is not the case if the baryon asymmetry is produced after or during the electroweak phase transition (see Refs. [河 and [5]).
} 
The lepton (or $B-L$ )-number violating operators (11) arise from the exchange of heavy Majorana neutrinos $N_{i}(i=1,2,3)$ [6]. Decays of these heavy neutrinos $N_{i}$ produce very naturally lepton (or $B-L$ ) asymmetry if $C$ and $C P$ are not conserved [8]. The produced lepton asymmetry is converted to baryon asymmetry through the "sphaleron" effects in the early universe [ [] ]. Therefore, the leptogenesis seems the most natural mechanism to account for the baryon asymmetry in the present universe.

There have been, so far, proposed various scenarios for the leptogenesis depending on production mechanisms of the heavy Majorana neutrinos $N_{i}$ [8 11]. Among them the most conventional is the thermal production of $N_{i}$ in the early universe. The detail analysis [9] shows that sufficient lepton asymmetry to explain the present baryon asymmetry can be obtained if the reheating temperature $T_{R}$ of inflation is $\mathcal{O}\left(10^{10}\right) \mathrm{GeV}$. However, when we consider the leptogenesis in the framework of supergravity, we have a cosmological gravitino problem: too many gravitinos are produced with $T_{R}=\mathcal{O}\left(10^{10}\right) \mathrm{GeV}$ to maintain the success of the big-bang nucleosynthesis (BBN) [12]. One way to solve the problem is to assume that the gravitino is the lightest supersymmetry (SUSY) particle of mass $m_{3 / 2} \simeq 10-100$ $\mathrm{GeV}$ [13]. Although this solution is a consistent and even interesting possibility, it is very important to find other leptogenesis scenarios consistent with the BBN in a wide region of the gravitino mass $m_{3 / 2} \simeq 100 \mathrm{GeV}-1 \mathrm{TeV}$.

In a recent article 15 we discussed leptogenesis via decays of the heavy Majorana neutrinos produced non-thermally in inflaton decays and found that the desired amount of lepton asymmetry is obtained for $T_{R} \simeq 10^{8} \mathrm{GeV}$. In this case the gravitino of mass $m_{3 / 2} \simeq 500 \mathrm{GeV}$ $1 \mathrm{TeV}$ becomes consistent with the BBN [12]. The purpose of this paper is to perform a detailed analysis on the leptogenesis, adopting a larger class of inflation models. We find that the required lepton asymmetry $n_{L} / s \simeq-10^{-10}$ (the ratio of the lepton-number density $n_{L}$ to the entropy density of the universe $s$ ) can be obtained even for $T_{R} \simeq 10^{6} \mathrm{GeV}$ in

\footnotetext{
${ }^{2}$ Another solution is to consider $m_{3 / 2} \lesssim 1 \mathrm{keV}$ [14]].
} 
some of the inflation models, and hence we conclude that there is no cosmological gravitino problem in the interesting wide region of the gravitino mass $m_{3 / 2} \simeq 100 \mathrm{GeV}-1 \mathrm{TeV}$. We assume SUSY and hence supergravity throughout this paper.

In Sec. [I] we review briefly the lepton-number production in the decays of the heavy Majorana neutrinos. The magnitude of the lepton-asymmetry parameter $\epsilon_{1}$ depends on various Yukawa coupling constants $\left(h_{\nu}\right)_{i j}$ and the mass ratios $M_{i} / M_{j}\left(M_{i}\right.$ denote masses of the $N_{i}$ ). For an estimation of $\epsilon_{1}$ we adopt the Froggatt-Nielsen model [16] for mass matrices of quarks and leptons including $N_{i}$, since it is very "successful" in explaining the observed mass hierarchies and mixing angles. The leptogenesis in a hybrid inflationary universe is discussed in Sec. III, where we consider two different types of SUSY hybrid inflation models. In Sec. IV we discuss the leptogenesis in a SUSY new inflation model. We also consider the case of a SUSY topological inflation in Sec. \. The last section is devoted to discussion and conclusions.

\section{LEPTON ASYMMETRY IN DECAYS OF HEAVY MAJORANA NEUTRINOS}

Before discussing lepton-number generation in decays of the heavy Majorana neutrinos, we briefly review the Froggatt-Nielsen (FN) model [16] for mass matrices of quarks and leptons. We adopt the FN model throughout this paper, since it seems the most attractive mechanism for explaining the observed hierarchies in the quark and charged lepton mass matrices. The model is based on a broken $U(1)_{F}$ family symmetry. A gauge-singlet field $\Phi$ carrying the $\mathrm{FN}$ charge $Q_{\Phi}=-1$ is assumed to have a vacuum-expectation value (vev) $\langle\Phi\rangle \neq$ 0 and then the Yukawa couplings of Higgs supermultiplets arise from nonrenormalizable interactions of $\Phi$ as

$$
W=g_{i j} \Phi^{Q_{i}+Q_{j}} \Psi_{i} \Psi_{j} H_{u(d)},
$$

where $Q_{i}$ are the FN charges of various supermultiplets $\Psi_{i}, g_{i j} \mathcal{O}(1)$ coupling constants. $H_{u}$ and $H_{d}$ are Higgs supermultiplets which couple to up-type and down-type quarks, respectively. Here and hereafter, we take the gravitational scale $M_{G} \simeq 2.4 \times 10^{18} \mathrm{GeV}$ equal to 


\begin{tabular}{|c|ccc|ccc|ccc|}
\hline \hline$\Psi_{i}$ & $l_{3}$ & $l_{2}$ & $l_{1}$ & $e_{3}^{c}$ & $e_{2}^{c}$ & $e_{1}^{c}$ & $N_{3}$ & $N_{2}$ & $N_{1}$ \\
\hline$Q_{i}$ & $a$ & $a$ & $a+1$ & 0 & 1 & 2 & $\mathrm{~b}$ & $\mathrm{c}$ & $\mathrm{d}$ \\
\hline \hline
\end{tabular}

TABLE I. The FN charges of various supermultiplets. We assume $a=0$ or 1 and $b \leq c<d$ in the text.

unity. The mass hierarchies for quarks and charged leptons are well explained in terms of their FN charges listed in Table @ with $\epsilon \equiv\langle\Phi\rangle \simeq 1 / 17$ [17]. The charges for $e_{i}^{c}$ are taken to be the same as those of up-type quarks assuming that they belong to the same 10's in the $S U(5)$ grand unified theory. The charge $a$ of the $l_{3}$ may be 0 or 1 (see Ref. [17] for details).

We apply the above mechanism to the heavy neutrino sector. Possible FN charges of

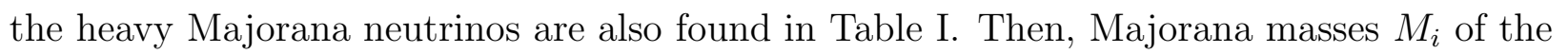
heavy neutrinos $N_{i}$ are given by

$$
M_{i} \simeq \epsilon^{2 Q_{i}} M_{0}
$$

where $Q_{i}$ denote the FN charges of $N_{i}$ and $M_{0}$ some mass scale of the $B-L$ breaking (see discussion in the next section). Furthermore, with the FN charges of the lepton doublets $l_{i}$ in Table [ the see-saw mechanism [6] induces a mass matrix for neutrinos [17, 18] as

$$
\left(m_{\nu}\right)_{i j} \simeq \epsilon^{2 a}\left(\begin{array}{ccc}
\epsilon^{2} & \epsilon & \epsilon \\
\epsilon & 1 & 1 \\
\epsilon & 1 & 1
\end{array}\right) \frac{\left\langle H_{u}\right\rangle^{2}}{M_{0}}
$$

As shown in Ref. [17], this mass matrix leads to a large $\nu_{\mu}-\nu_{\tau}$ mixing angle which is consistent with the atmospheric neutrino oscillation observed in the Superkamiokande experiments [7]. From the observed value of the mass squared difference for neutrinos $\nu_{i}(i=1,2,3)$, $m_{\nu_{3}}^{2}-m_{\nu_{2}}^{2}=(0.5-6) \times 10^{-3} \mathrm{eV}^{2}[7]$, we find the mass of the heaviest neutrino $\nu_{3}$ as $m_{\nu_{3}} \simeq$ 
$(2-8) \times 10^{-2} \mathrm{eV}$, provided a mass hierarchy, $m_{\nu_{3}} \gg m_{\nu_{2}}$. Then, we may derive?

$$
M_{0} \simeq \epsilon^{2 a} \frac{\left\langle H_{u}\right\rangle^{2}}{m_{\nu_{3}}} \simeq \epsilon^{2 a}(0.3-1) \times 10^{15} \mathrm{GeV} \simeq\left\{\begin{array}{ll}
(0.3-1) \times 10^{15} \mathrm{GeV} & \text { for } a=0 \\
(1-3) \times 10^{12} \mathrm{GeV} & \text { for } a=1
\end{array} .\right.
$$

We are now at the point to see generation of the lepton asymmetry in decays of the heavy Majorana neutrinos. Because the decays of the heavy Majorana neutrinos $N_{i}$ into lepton $l_{j}$ and Higgs $H_{u}$ doublets have the following two distinct decay channels;

$$
\begin{aligned}
& N_{i} \rightarrow H_{u}+l_{j} \\
& N_{i} \rightarrow \overline{H_{u}}+\overline{l_{j}},
\end{aligned}
$$

the interference between decay amplitudes of tree and one-loop diagrams results in the lepton-number asymmetry if $C P$ is not conserved [8]. Here and hereafter, $N_{i}, l_{j}\left(\overline{l_{j}}\right)$ and $H_{u}\left(\overline{H_{u}}\right)$ denote fermionic or scalar (bosonic) components of corresponding supermultiplets unless we explicitly distinguish them. We consider only the $N_{1}$ decay, provided that the mass $M_{1}$ is much smaller than the others $\left(M_{1} \ll M_{2}, M_{3}\right.$, i.e., $\left.d>b, c\right)$. From Eqs. (3) and (5) the $M_{1}$ is evaluated with the FN charge $d$ as

$$
M_{1} \simeq \epsilon^{2 d} M_{0} \simeq\left\{\begin{array}{ll}
(1-3) \times 10^{12} \mathrm{GeV} & \text { for } a+d=1 \\
(0.3-1) \times 10^{10} \mathrm{GeV} & \text { for } a+d=2
\end{array} .\right.
$$

In the following analysis, we only consider the case $a+d=1$ or $a+d=2$. The total decay width of the $N_{1}, \Gamma_{N_{1}}$, is given by

$$
\Gamma_{N 1} \simeq \frac{1}{4 \pi} \epsilon^{2(a+d)} M_{1} \simeq\left\{\begin{array}{ll}
(0.3-1) \times 10^{9} \mathrm{GeV} & \text { for } a+d=1 \\
(0.3-1) \times 10^{4} \mathrm{GeV} & \text { for } a+d=2
\end{array} .\right.
$$

The lepton asymmetry produced in the $N_{1}$ decay is represented by a parameter $\epsilon_{1}$, which is calculated as [19,20]

\footnotetext{
${ }^{3}$ We assume, in this paper, $\tan \beta \equiv\left\langle H_{u}\right\rangle /\left\langle H_{d}\right\rangle \simeq 1$ and hence $\left\langle H_{u}\right\rangle \simeq 123 \mathrm{GeV}$. Even if one takes a larger $\tan \beta \simeq 50$, the discussion in the text does not change too much (i.e., the obtained lepton asymmetry is reduced only by factor 2 ).
} 


$$
\begin{aligned}
\epsilon_{1} & \equiv \frac{\Gamma\left(N_{1} \rightarrow H_{u}+l\right)-\Gamma\left(N_{1} \rightarrow \overline{H_{u}}+\bar{l}\right)}{\Gamma\left(N_{1} \rightarrow H_{u}+l\right)+\Gamma\left(N_{1} \rightarrow \overline{H_{u}}+\bar{l}\right)} \\
& =-\frac{3}{16 \pi\left(h_{\nu} h_{\nu}^{\dagger}\right)_{11}}\left[\operatorname{Im}\left(h_{\nu} h_{\nu}^{\dagger}\right)_{13}^{2} \frac{M_{1}}{M_{3}}+\operatorname{Im}\left(h_{\nu} h_{\nu}^{\dagger}\right)_{12}^{2} \frac{M_{1}}{M_{2}}\right] .
\end{aligned}
$$

Here we have taken a basis where the mass matrix for $N_{i}$ is diagonal and the Yukawa couplings $\left(h_{\nu}\right)_{i j}$ are defined in the superpotential as $W=\left(h_{\nu}\right)_{i j} N_{i} l_{j} H_{u}$. We have included both of one-loop vertex and self-energy corrections [20.7.

The FN model [see Eqs. (2) and (3)] allows us to rewrite the lepton-asymmetry parameter $\epsilon_{1}$ in Eq. (9) by using an effective $C P$-violating phase $\delta_{\text {eff }}$ as [15]

$$
\begin{aligned}
\epsilon_{1} & \simeq \frac{3 \delta_{\mathrm{eff}}}{16 \pi\left(h_{\nu} h_{\nu}^{\dagger}\right)_{11}}\left|\left(h_{\nu} h_{\nu}^{\dagger}\right)_{13}^{2}\right| \frac{M_{1}}{M_{3}} \simeq \frac{3 \delta_{\mathrm{eff}}}{16 \pi} \epsilon^{2(a+d)} \\
& \simeq \frac{3 \delta_{\mathrm{eff}}}{16 \pi} \frac{m_{\nu_{3}} M_{1}}{\left\langle H_{u}\right\rangle^{2}}
\end{aligned}
$$

where we have also used Eq. (四) to derive the last equation (11). Notice that the asymmetry parameter $\epsilon_{1}$ depends only on the FN charge $(a+d)$. Taking the maximum $C P$ violating phase $\left|\delta_{\text {eff }}\right| \simeq 1$, we obtain 18

$$
\begin{gathered}
\epsilon_{1} \simeq\left\{\begin{array}{l}
-2 \times 10^{-4} \text { for } a+d=1 \\
-7 \times 10^{-7} \text { for } a+d=2
\end{array},\right. \\
\simeq-(1-3) \times 10^{-6}\left(\frac{M_{1}}{10^{10} \mathrm{GeV}}\right) .
\end{gathered}
$$

Let us now assume that the heavy neutrinos $N_{1}$ are produced in inflaton $\varphi$ decay, which leads to a constraint on the inflaton mass $m_{\varphi}$ as

$$
m_{\varphi}>2 M_{1}
$$

and calculate a net lepton-number asymmetry created via the decays of $N_{1}$. First of all, we restrict ourselves only to inflation models with reheating temperatures $T_{R}$ being lower

\footnotetext{
${ }^{4} N_{1}$ in Eq. (9) denotes fermionic or scalar component of the supermultiplet $N_{1}$. Thus, the lepton-asymmetry parameter $\epsilon_{1}$ in the decay of scalar component $\widetilde{N}_{1}$ is the same as that in the decay of fermionic component $N_{1}$.
} 
than $10^{8} \mathrm{GeV}$ to avoid overproduction of the gravitinos. In this case the produced heavy Majorana neutrinos $N_{1}$ are always out of thermal equilibrium since $M_{1} \gtrsim 10 T_{R}$ [see Eq. (7) and discussions in subsequent sections] and the $N_{1}$ behave like frozen-out, relativistic particles with the energy of $E_{N_{1}}=m_{\varphi} / 2$. Furthermore, the $N_{1}$ decay immediately after produced in the inflaton $\varphi$ decay as we will see in the subsequent sections. With these conditions we may easily estimate lepton-to-entropy ratio as 10

$$
\begin{aligned}
\frac{n_{L}}{s} & \simeq \frac{3}{2} \epsilon_{1} B_{r} \frac{T_{R}}{m_{\varphi}} \\
& \simeq-(1-3) \times 10^{-6} B_{r}\left(\frac{T_{R}}{10^{10} \mathrm{GeV}}\right)\left(\frac{M_{1}}{m_{\varphi}}\right),
\end{aligned}
$$

where $B_{r}$ is the branching ratio of the inflaton decay $\varphi \rightarrow N_{1} N_{1}$ channel. Notice that the reheating temperature $T_{R}$ is bounded from below, $T_{R} \gtrsim 10^{5} \mathrm{GeV}$, otherwise the produced lepton asymmetry is too small to explain the baryon number in the present universe.

The lepton asymmetry in Eq. (15) is converted to the baryon asymmetry through the "sphaleron" effects which is given by

$$
\frac{n_{B}}{s} \simeq a \frac{n_{L}}{s}
$$

with $a \simeq-8 / 23$ [21] in the minimal SUSY standard model. To explain the observed baryon asymmetry

$$
\frac{n_{B}}{s} \simeq(0.1-1) \times 10^{-10}
$$

we should have the lepton asymmetry

$$
\frac{n_{L}}{s} \simeq-(0.3-3) \times 10^{-10}
$$

In the subsequent sections [II, IV and V we will examine whether the required lepton asymmetry Eq. (18) is obtained with low enough reheating temperature of $T_{R} \lesssim 10^{8} \mathrm{GeV}$ to avoid the cosmological gravitino problem. For practical calculations we will use three types of SUSY models for hybrid, new and topological inflations. 


\section{LEPTOGENESIS IN HYBRID INFLATION}

In this section we perform a detailed analysis on hybrid inflation models and examine whether they can provide us with sufficient lepton asymmetry to account for the baryon asymmetry in the present universe, avoiding overproduction of the gravitinos to maintain the success of the BBN. Before discussing hybrid inflation models, we show first a particlephysics model for the heavy Majorana neutrinos $N_{i}$.

A simple extension of the SUSY standard electroweak gauge theory is given by considering the gauged $B-L$ symmetry, in which right-handed neutrinos $N_{i}$ are necessary to cancel $B-L$ gauge anomaly. We introduce standard-model gauge-singlet supermultiplets $\Psi(x, \theta)$ and $\bar{\Psi}(x, \theta)$ carrying $B-L$ charges +2 and -2 , respectively, and suppose that the $B-L$ symmetry is spontaneously broken by the condensations $\langle\Psi\rangle=\langle\bar{\Psi}\rangle$ at high energies. heavy neutrinos $N_{i}$ acquire Majorana masses through the following superpotential:[0

$$
W=\frac{1}{2} g_{i} N_{i} N_{i} \Psi
$$

Here we have taken a basis where the above Yukawa coupling matrix $g$ is diagonal. We assume that $\Psi$ and $\bar{\Psi}$ have zero FN charges and then the $M_{0}$ in Eq. (5) is

$$
M_{0} \simeq\langle\Psi\rangle=\langle\bar{\Psi}\rangle
$$

Namely, the $B-L$ gauge symmetry is broken down at the scale about $10^{15} \mathrm{GeV}$ or $10^{12}$ $\mathrm{GeV}$, generating large Majorana masses of the right-handed neutrinos $N_{i}$ [see Eq. (5)].]

A superpotential causing the $B-L$ breaking is given by

$$
W=-\mu^{2} \phi+\lambda \phi \Psi \bar{\Psi}
$$

\footnotetext{
${ }^{5}$ We always take $\Psi=\bar{\Psi}$ to satisfy the $D$-term flatness condition of the $U(1)_{B-L}$.

${ }^{6}$ Lazarides has discussed [22] the leptogenesis in the hybrid inflation assuming the nonrenormalizable superpotential $W=\left(g_{i}^{\prime} / 2\right) N_{i} N_{i} \Psi \Psi$, where the $B-L$ charge of $\Psi$ is taken as +1 .

7 This model is easily embedded in the $S O(10)$ grand unified theory.
} 
where $\phi(x, \theta)$ is a gauge-singlet supermultiplet, $\lambda$ a coupling constant and $\mu$ a dimensional mass parameter. Notice that this superpotential possesses a $U(1) R$-symmetry where the $\phi$ and $\Psi \bar{\Psi}$ have $U(1)_{R}$ charges 2 and 0 , respectively. The potential for scalar components of the supermultiplets $\phi(x, \theta), \Psi(x, \theta)$ and $\bar{\Psi}(x, \theta)$ is given by, in supergravity,

$$
V=e^{K}\left\{\left(\frac{\partial^{2} K}{\partial z_{I} \partial z_{J}^{*}}\right)^{-1} D_{z_{I}} W D_{z_{J}^{*}} W^{*}-3|W|^{2}\right\}+D \text {-terms }
$$

where

$$
D_{z_{I}} W=\frac{\partial W}{\partial z_{I}}+\frac{\partial K}{\partial z_{I}} W
$$

and $z_{I}$ denote scalar components $\phi, \Psi$ and $\bar{\Psi}$ of the corresponding supermultiplets. Here we have assumed the $R$-invariant Kähler potential for $\phi, \Psi$ and $\bar{\Psi}$ as

$$
K=|\phi|^{2}+|\Psi|^{2}+|\bar{\Psi}|^{2}+\frac{\kappa_{1}}{4}|\phi|^{4}+\cdots
$$

where the ellipsis denotes higher-order terms which we neglect in the present analysis. Then, we have a SUSY-invariant vacuum?

$$
\begin{aligned}
& \langle\Psi\rangle=\langle\bar{\Psi}\rangle=\sqrt{\frac{\mu^{2}}{\lambda}}, \\
& \langle\phi\rangle=0 .
\end{aligned}
$$

It is quite interesting to observe that the superpotential (21) is nothing but one proposed in Refs. 22,24 for a SUSY hybrid inflation model. The real part of $\phi$ is identified with the inflaton field $\varphi / \sqrt{2}$. Furthermore, the potential is minimized at $\Psi=\bar{\Psi}=0$ when $\varphi$ is larger than $\varphi_{c} \equiv \sqrt{2 \mu^{2} / \lambda}$, and hybrid inflation occurs for $\varphi_{c}<\varphi \lesssim 1$ and $k \equiv-\kappa_{1} \geq 0$. Including one-loop corrections [23] the potential for the inflaton $\varphi$ is given by, for $\varphi>\varphi_{c}$,

$$
\begin{aligned}
V \simeq & \mu^{4}+\frac{k}{2} \mu^{4} \varphi^{2}+\frac{1}{16}\left(4 k^{2}+7 k+2\right) \mu^{4} \varphi^{4} \\
& +\frac{\lambda^{4}}{128 \pi^{2}}\left[2 \varphi_{c}^{4} \ln \left(\frac{\lambda^{2} \varphi^{2}}{2 \Lambda^{2}}\right)+\left(\varphi^{2}-\varphi_{c}^{2}\right)^{2} \ln \left(1-\frac{\varphi_{c}^{2}}{\varphi^{2}}\right)+\left(\varphi^{2}+\varphi_{c}^{2}\right)^{2} \ln \left(1+\frac{\varphi_{c}^{2}}{\varphi^{2}}\right)\right]
\end{aligned}
$$

\footnotetext{
${ }^{8}$ We take a basis where $\mu^{2}$ and $\lambda$ are real and positive by using the phase rotations of $\phi$ and $\Psi \bar{\Psi}$. Using the $B-L$ rotation we choose $\langle\Psi\rangle$ to be real and positive.
} 
where $\Lambda$ denotes the renormalization scale. Here we have included in the inflaton potential $\mu^{4} \varphi^{4}$ terms induced by the Kähler potential (24) since the initial value of the inflaton field is close to 1. The mass of the inflaton $\varphi$ in the true vacuum Eq. (25) is estimated as

$$
m_{\varphi} \simeq \sqrt{2 \lambda} \mu \text {. }
$$

Let us now discuss the inflation dynamics. The slow-roll conditions for inflation are given by [25],

$$
\begin{aligned}
& \frac{1}{2}\left(\frac{V^{\prime}}{V}\right)^{2}<1, \\
& \left|\frac{V^{\prime \prime}}{V}\right|<1,
\end{aligned}
$$

where the prime denotes the derivative with the inflaton field. These conditions are satisfied when $\varphi>\varphi_{c}, \lambda<1$ and $1>k \geq 0$. Therefore, while the inflaton $\varphi$ rolls down along the potential (26) from $\varphi_{I}\left(1 \gtrsim \varphi_{I}>\varphi_{c}\right)$ to $\varphi_{c}$, the vacuum energy $\mu^{4}$ of the potential dominates the energy of the universe and hence the hybrid inflation takes place [26].

After the inflation ends, the vacuum energy is transferred into the energies of the coherent oscillations of the inflaton $\varphi$ and the scalar field $\Sigma=(\Psi+\bar{\Psi}) / \sqrt{2}$. The radiations of the universe are produced by the decays of the $\varphi$ and/or $\Sigma$ field and the universe is reheated at the temperature $T_{R}$. In order to estimate the reheating temperature $T_{R}$ we have to know total decay rates of these scalar fields. Through the interactions in the superpotentials (19) and (21), the inflaton $\varphi$ decays into scalar components $\widetilde{N}_{1}$ of the $N_{1}$ supermultiplet, if kinematically allowed, with the rate

$$
\Gamma_{\varphi} \simeq \Gamma\left(\varphi \rightarrow \widetilde{N}_{1} \widetilde{N}_{1}\right)=\frac{1}{64 \pi} \frac{M_{1}^{2} m_{\varphi}}{\langle\Psi\rangle^{2}}\left(1-\frac{4 M_{1}^{2}}{m_{\varphi}^{2}}\right)^{1 / 2} .
$$

The $\Sigma$ field decays into scalar and fermionic components of the $N_{1}$ with

\footnotetext{
${ }^{9}$ We have neglected the $\mu^{4} \varphi^{4}$ terms coming from the higher order interactions in the Kähler potential (e.g., $\left.K=\kappa^{\prime}|\phi|^{6}\right)$.
} 


$$
\begin{aligned}
& \Gamma\left(\Sigma \rightarrow \widetilde{N}_{1} \widetilde{N}_{1}\right)=\frac{1}{16 \pi} \frac{M_{1}^{4}}{\langle\Psi\rangle^{2} m_{\Sigma}}\left(1-\frac{4 M_{1}^{2}}{m_{\Sigma}^{2}}\right)^{1 / 2}, \\
& \Gamma\left(\Sigma \rightarrow N_{1} N_{1}\right)=\frac{1}{64 \pi} \frac{M_{1}^{2} m_{\Sigma}}{\langle\Psi\rangle^{2}}\left(1-\frac{4 M_{1}^{2}}{m_{\Sigma}^{2}}\right)^{3 / 2}
\end{aligned}
$$

if $m_{\Sigma}>2 M_{1}$. Notice that the inflaton $\varphi$ forms a massive supermultiplet together with the $\Sigma$ field in the vacuum (25) and the mass of the $\Sigma$ field is equal to the inflaton mass $\left(m_{\Sigma}=m_{\varphi} \simeq \sqrt{2 \lambda} \mu\right)$.

Since the $\Sigma$ field has a non-zero vev, the $\Sigma$ decays also through nonrenormalizable interactions in the Kähler potential,एण

$$
K=\sum_{i} c_{i}|\Sigma|^{2}\left|\psi_{i}\right|^{2}
$$

where $\psi_{i}$ denote supermultiplets of the SUSY standard-model particles including the heavy Majorana neutrinos, and $c_{i}$ coupling constants of order unity. Then the decay rate by these interactions is estimated as

$$
\Gamma\left(\Sigma \rightarrow \psi_{i} \overline{\psi_{i}}\right) \simeq \frac{1}{8 \pi} C\langle\Psi\rangle^{2} m_{\Sigma}^{3}
$$

where $C=\sqrt{\sum c_{i}^{2}}$ is a parameter of order unity. In the following analysis we take $C=1$. Comparing the rate (34) with Eqs. (31) and (32), we easily see that the total decay rate of the $\Sigma$ field is determined by Eq. (32) since $\Gamma_{\Sigma} \simeq \Gamma\left(\Sigma \rightarrow N_{1} N_{1}\right)>\Gamma\left(\Sigma \rightarrow \widetilde{N}_{1} \widetilde{N}_{1}\right)$ for $m_{\Sigma}>2 M_{1}$

We assume $m_{\varphi}=m_{\Sigma}>2 M_{1}$ as discussed in the previous section [see Eq. (14)], and hence the decays of the inflaton $\varphi$ and the $\Sigma$ field take place at the almost same time because of $\Gamma_{\varphi} \simeq \Gamma_{\Sigma}$. Thus, the reheating temperature $T_{R}$ is estimated by the total decay width of the $\Sigma$ field as

$$
T_{R} \simeq 0.46 \sqrt{\Gamma_{\Sigma}}
$$

10 The decays of the $\Sigma$ field through the $B-L$ gauge-multiplet exchanges are negligible. 
The total decay rate of the $\Sigma$ field is much smaller than the decay rate of the $N_{1}$ [see Eq. (8)] and the $N_{1}$ decays immediately after produced in the $\Sigma$ decay. This guarantees the validity of the formula of the lepton asymmetry given by Eq. (15). Here it should be noted that the branching ratio of the $\Sigma$ (inflaton $\varphi$ ) decay into two $N_{1}\left(\widetilde{N_{1}}\right)$ in Eq. (15) is $B_{r} \simeq 1$.

The above hybrid inflation must explain the following two observations: (i) the $e$-hold number $N_{e}$ of the present horizon and (ii) the density fluctuations observed by the cosmic background explorer (COBE) satellite. While the inflaton $\varphi$ rolls down along the potential (26) from $\varphi_{N_{e}}$ to $\varphi_{c}$, the scale factor of the universe increases by $e^{N_{e}}$. This $e$-fold number $N_{e}$ is given by

$$
N_{e} \simeq \int_{\varphi_{c}}^{\varphi_{N_{e}}} d \varphi \frac{V(\varphi)}{V^{\prime}(\varphi)}
$$

In order to explain the present horizon scale, the $e$-fold number should be

$$
N_{e}=67+\frac{1}{3} \ln \left(H_{I} T_{R}\right)
$$

where $H_{I} \simeq \mu^{2} / \sqrt{3}$ denotes the Hubble parameter during the inflation. Furthermore, the amplitude of the primordial density fluctuations $\delta \rho / \rho$ predicted by the hybrid inflation,

$$
\frac{\delta \rho}{\rho} \simeq \frac{1}{5 \sqrt{3} \pi} \frac{V^{3 / 2}\left(\varphi_{N_{e}}\right)}{\left|V^{\prime}\left(\varphi_{N_{e}}\right)\right|}
$$

should be normalized by the data on anisotropies of the cosmic microwave background radiation (CMBR) observed by the COBE satellite [27], which gives

$$
\frac{V^{3 / 2}\left(\varphi_{N_{e}}\right)}{\left|V^{\prime}\left(\varphi_{N_{e}}\right)\right|} \simeq 5.3 \times 10^{-4} .
$$

From Eqs. (37) and (39) the scale $\mu$ of the hybrid inflation is determined for given $\lambda$ and $k$. We have performed numerical calculations and the obtained $\mu$ is shown in Fig. 11. (Note that we find no sizable difference in the scale $\mu$ for between $a+d=1$ and $a+d=2$.) We should exclude the region where $\varphi_{N_{e}} \gtrsim 1$, because our effective treatment of the inflaton potential (26) becomes invalid in that region. The vev of $\Psi$ and the inflaton mass $m_{\varphi}$ are found in Figs. 2 and 3, respectively. It is interesting that as shown in Fig. 2 the scale of 


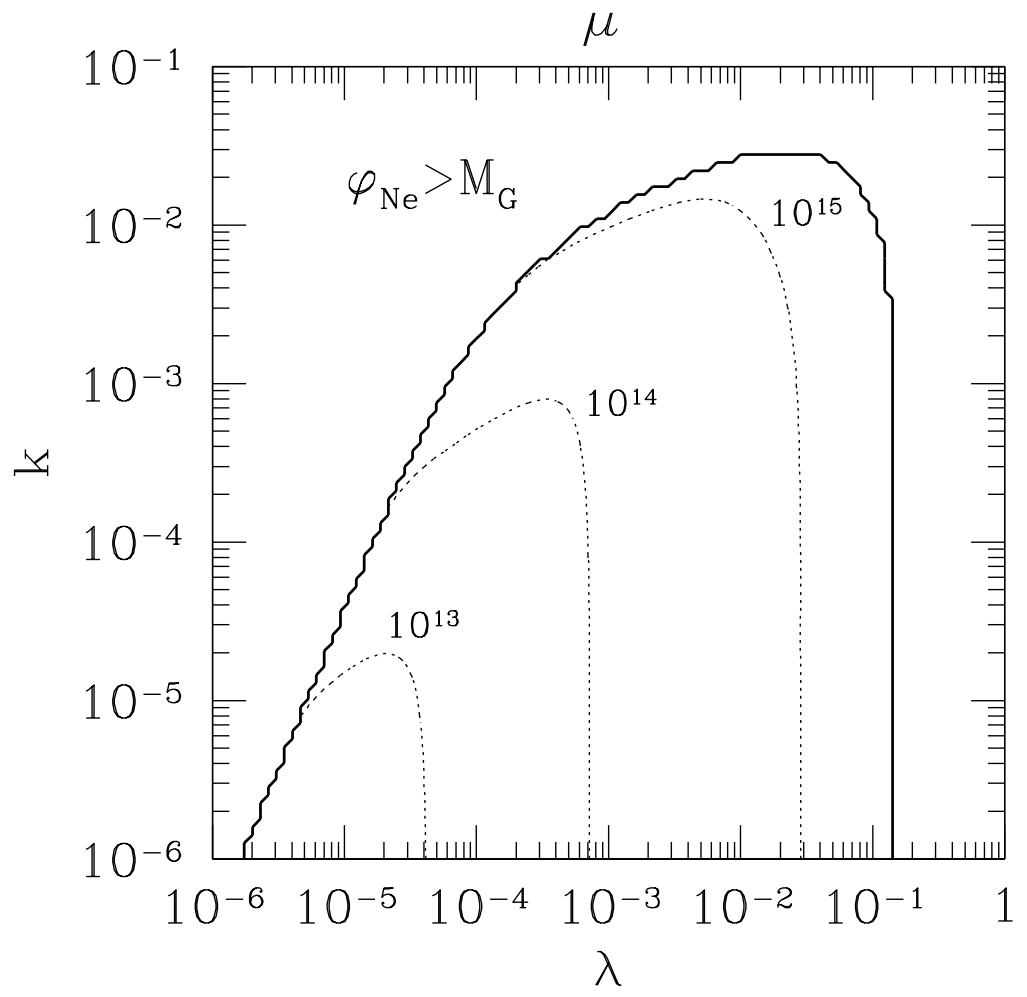

FIG. 1. The contour lines of the scale $\mu$ in the hybrid inflation model. The contour lines are all shown by the dotted lines and corresponding values of $\mu$ are also represented in unit of $\mathrm{GeV}$. The upper bound on $k$ from the requirement $\varphi_{N_{e}}<M_{G}$ is shown by the thick solid line.

the $B-L$ breaking is predicted as $\langle\Psi\rangle \simeq(1-5) \times 10^{15} \mathrm{GeV}$ in a wide parameter region, $10^{-6} \lesssim \lambda \lesssim 10^{-2}$ and $k \lesssim 10^{-3}$, which is very consistent with $M_{0} \simeq 10^{15} \mathrm{GeV}$ (i.e., $a=0$ ) derived from the observed neutrino mass [see Eq. (5)]. On the other hand, the lower value of the $B-L$ breaking scale of $M_{0} \simeq 10^{12} \mathrm{GeV}(a=1)$ cannot be obtained in the present hybrid inflation model.

The reheating temperature $T_{R}$ depends on the mass of the lightest Majorana neutrino, $M_{1}$ [see Eqs. (32) and (35)]. We show the $T_{R}$ in Figs. 1 and 5 for the case $M_{1} \simeq 10^{12}$ $\mathrm{GeV}(a+d=1)$ and for the case $M_{1} \simeq 3 \times 10^{9} \mathrm{GeV}(a+d=2)$, respectively. $\quad$ It is found that for the region of the inflaton mass $m_{\varphi}=m_{\Sigma} \gg 2 M_{1}$ the reheating temperature $T_{R} \lesssim 10^{8} \mathrm{GeV}$, which is required to avoid the cosmological gravitino problem, is obtained 


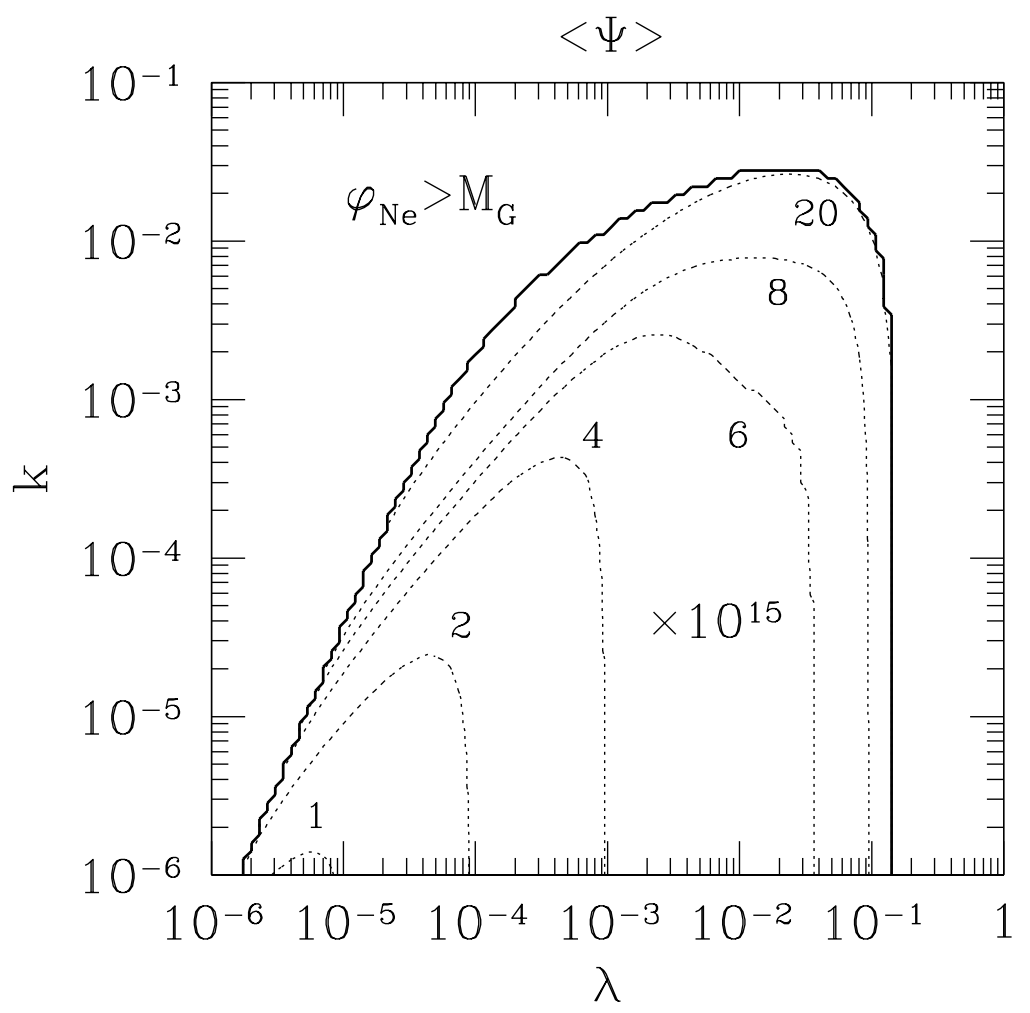

FIG. 2. The contour lines of the vev $\langle\Psi\rangle$ in the hybrid inflation model. The contour lines are all shown by the dotted lines and corresponding values of $\langle\Psi\rangle$ are also represented in unit of $10^{15}$ $\mathrm{GeV}$. The upper bound on $k$ from the requirement $\varphi_{N_{e}}<M_{G}$ is shown by the thick solid line.

only for the case $M_{1} \simeq 3 \times 10^{9} \mathrm{GeV}(a+d=2)$. Notice that for the region $m_{\varphi} \leq 2 M_{1}$ the reheating temperature is determined by the decay width (34) and the desired low reheating temperature is obtained even for the case of $M_{1} \simeq 10^{12} \mathrm{GeV}(a+d=1)$. However, such cases are not interesting since the $N_{1}$ are not produced in the $\varphi$ and $\Sigma$ decays and leptogenesis does not take place.

We now examine whether the leptogenesis works well or not in the above hybrid inflation model. Since the heavy Majorana neutrinos $N_{1}$ are produced in the decays of the inflaton $\varphi$ and the $\Sigma$ field, the mass of the inflaton should satisfy Eq. (14). As derived in Sec. II, the ratio of the produced lepton number to the entropy, $n_{L} / s$, is given by Eq. (15). Notice that $B_{r} \simeq 1$ in the present hybrid inflation. We show in Figs. 6 and 7 the $n_{L} / s$ for the 


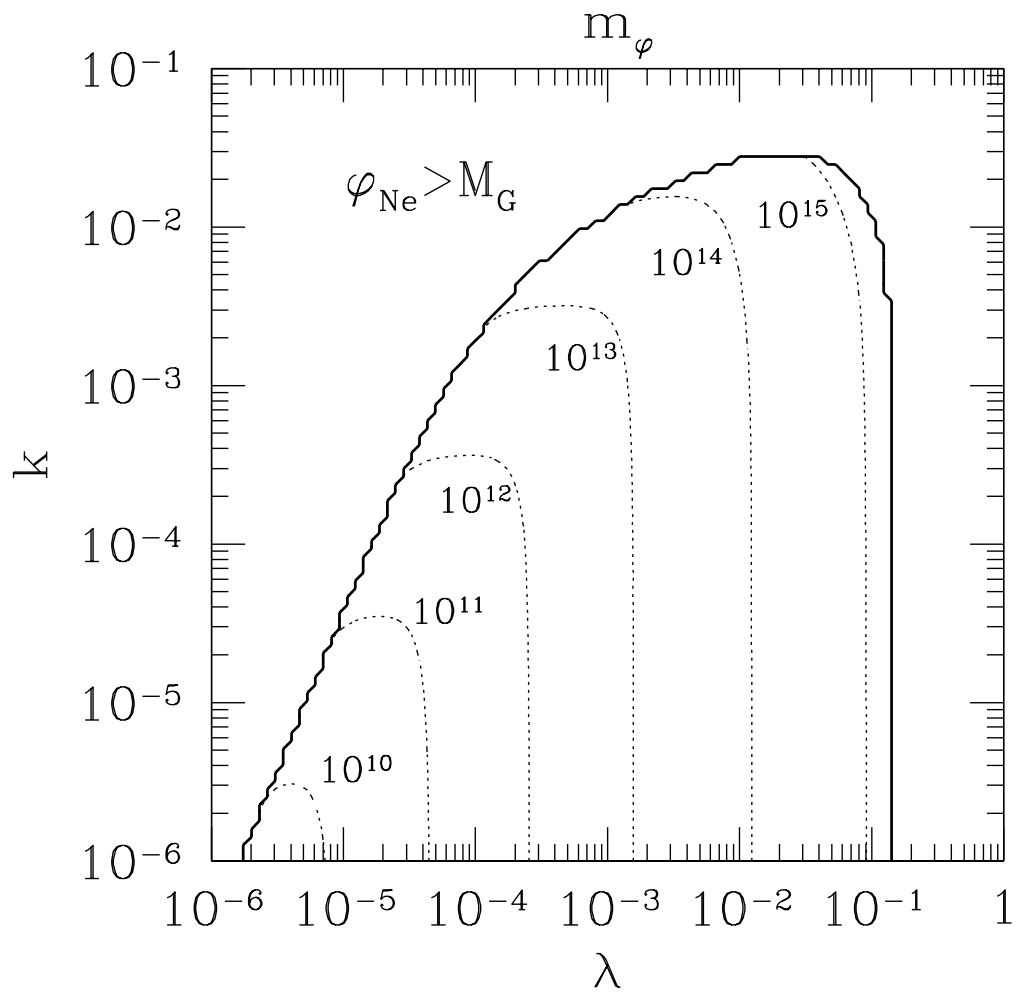

FIG. 3. The contour lines of the inflaton mass $m_{\varphi}$ in the hybrid inflation model. The contour lines are all shown by the dotted lines and corresponding values of $m_{\varphi}$ are also represented in unit of $\mathrm{GeV}$. The upper bound on $k$ from the requirement $\varphi_{N_{e}}<M_{G}$ is shown by the thick solid line.

cases $a+d=1$ and $a+d=2$, respectively. First, we consider the case of $M_{1} \simeq 10^{12} \mathrm{GeV}$ $(a+d=1)$. We find from Fig. 6 that the lepton asymmetry enough to explain the present baryon asymmetry can be generated in a wide parameter region. However, we have too high reheating temperature of $T_{R} \simeq 10^{9}-10^{12} \mathrm{GeV}$ as mentioned before. $\square$ (This parameter region may be interesting when some entropy production of order $10^{3}-10^{4}$ takes place at low

\footnotetext{
${ }^{11}$ For the higher reheating temperature of $T_{R} \gtrsim 10^{11} \mathrm{GeV}$, the formula of the lepton asymmetry (15) should not be verified, since $M_{1} / T_{R} \lesssim 10$ and the inverse decay process of the $N_{1}$ might be effective. However, this does not affect our conclusion, because we concentrate ourselves only to the case $T_{R} \lesssim 10^{8} \mathrm{GeV}$.
} 


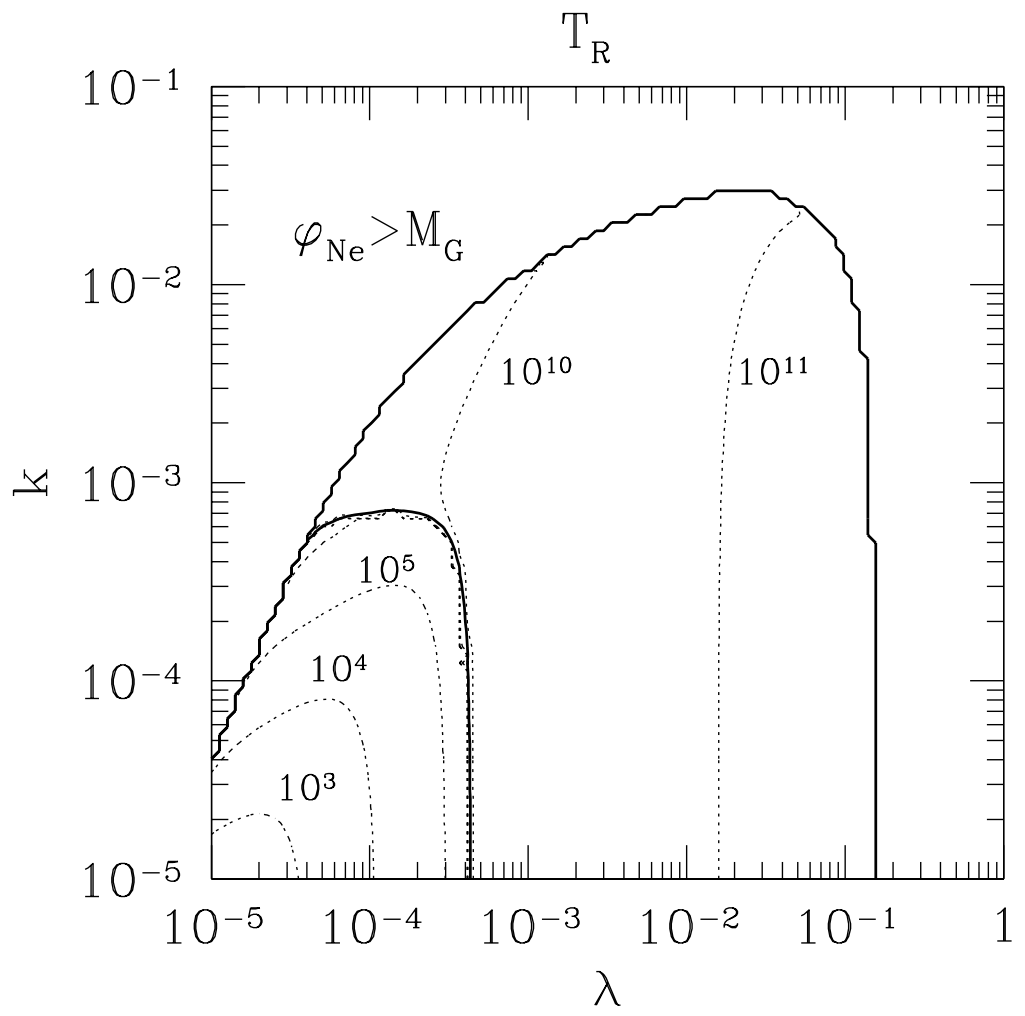

FIG. 4. The contour lines of the reheating temperature $T_{R}$ in the hybrid inflation model for the case $M_{1} \simeq 10^{12} \mathrm{GeV}(a+d=1)$. The contour lines are all shown by the dotted lines and corresponding values of $T_{R}$ are also represented in unit of $\mathrm{GeV}$. The upper bound on $k$ from the requirement $\varphi_{N_{e}}<M_{G}$ is shown by the thick solid line. The $k$ yielding $m_{\varphi}=m_{\Sigma}=2 M_{1}$ is also shown by the thick solid line.

energies.) Therefore, only the small region of $m_{\Sigma} \simeq 2 M_{1}$, where the reheating temperature becomes smaller as $T_{R} \simeq 10^{6}-10^{8} \mathrm{GeV}$ due to the phase volume suppression, ${ }^{\mp}$ is free from the cosmological gravitino problem. In this narrow region, we obtain the required lepton asymmetry of $n_{L} / s \simeq-(0.3-3) \times 10^{-10}$ [see Eq. (18)] to account for the present baryon asymmetry.

Next, we consider the case of $M_{1} \simeq 3 \times 10^{9} \mathrm{GeV}(a+d=2)$. From Fig. 7 it is found

\footnotetext{
${ }^{12}$ In this case, we should include the decay rates (31) and (34) in estimating $T_{R}$.
} 


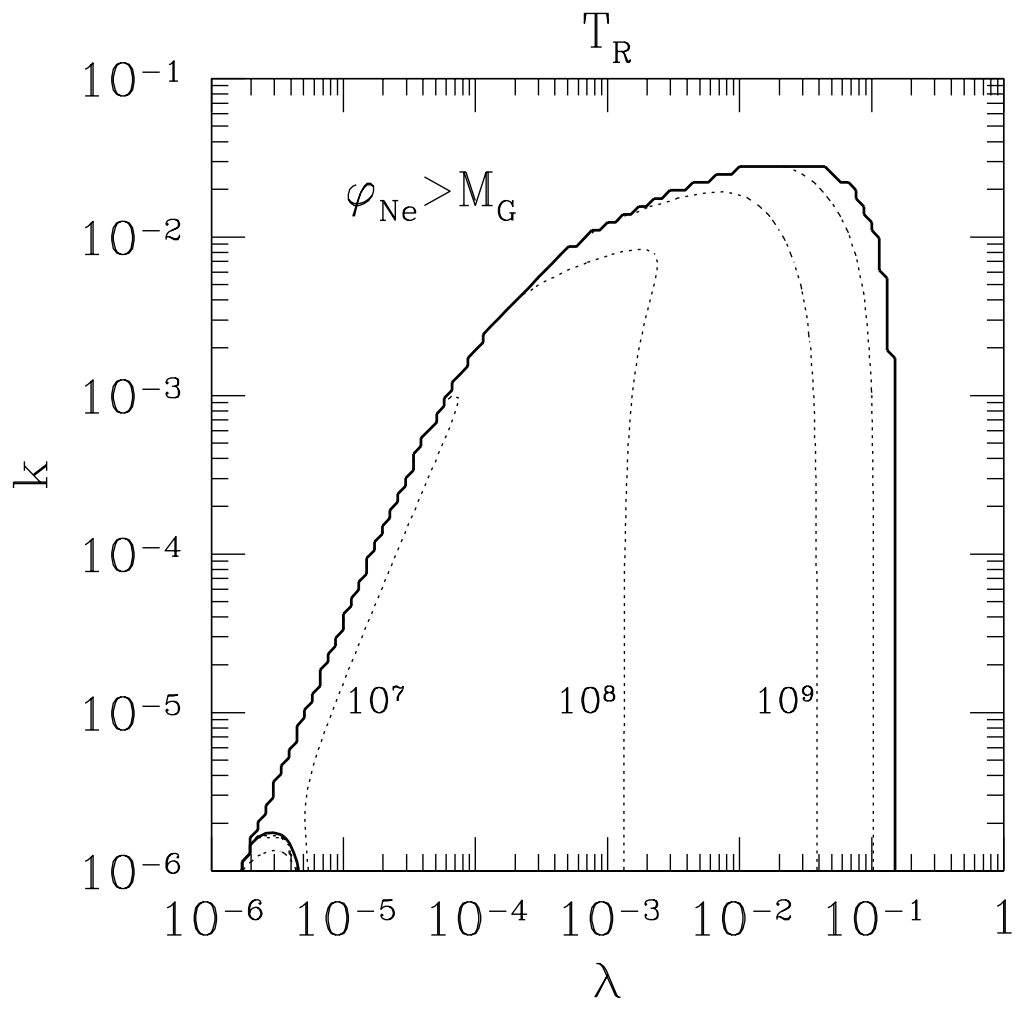

FIG. 5. The contour lines of the reheating temperature $T_{R}$ in the hybrid inflation model for the case $M_{1} \simeq 3 \times 10^{9} \mathrm{GeV}(a+d=2)$. The contour lines are all shown by the dotted lines and corresponding values of $T_{R}$ are also represented in unit of $\mathrm{GeV}$. The upper bound on $k$ from the requirement $\varphi_{N_{e}}<M_{G}$ is shown by the thick solid line. The $k$ yielding $m_{\varphi}=m_{\Sigma}=2 M_{1}$ is also shown by the thick solid line.

that the required lepton asymmetry of $n_{L} / s \simeq-10^{-10}$ as well as the low enough reheating temperature of $T_{R} \simeq 10^{7}-10^{8} \mathrm{GeV}$ are naturally offered in the region of $k \lesssim 10^{-3}$ and $\lambda \simeq 10^{-6}-10^{-3}$. Therefore, we conclude that the hybrid inflation with $M_{1} \simeq 3 \times 10^{9}$ $\mathrm{GeV}$ can produce a sufficient lepton asymmetry giving a reheating temperature low enough to solve the cosmological gravitino problem [15]. However, the reheating temperature of

${ }^{13}$ In the region of $T_{R} \lesssim 10^{8} \mathrm{GeV}$ the formula for the lepton asymmetry Eq. (15) is justified since $M_{1} \gtrsim 10 T_{R}$. 
$T_{R} \simeq 10^{6} \mathrm{GeV}$ which is required to avoid the cosmological difficulty for the gravitino of mass $m_{3 / 2} \simeq 100-500 \mathrm{GeV}$, is achieved only in the narrow parameter region of $m_{\varphi}=m_{\Sigma} \simeq 2 M_{1}$ as in the previous case $(a+d=1)$. 


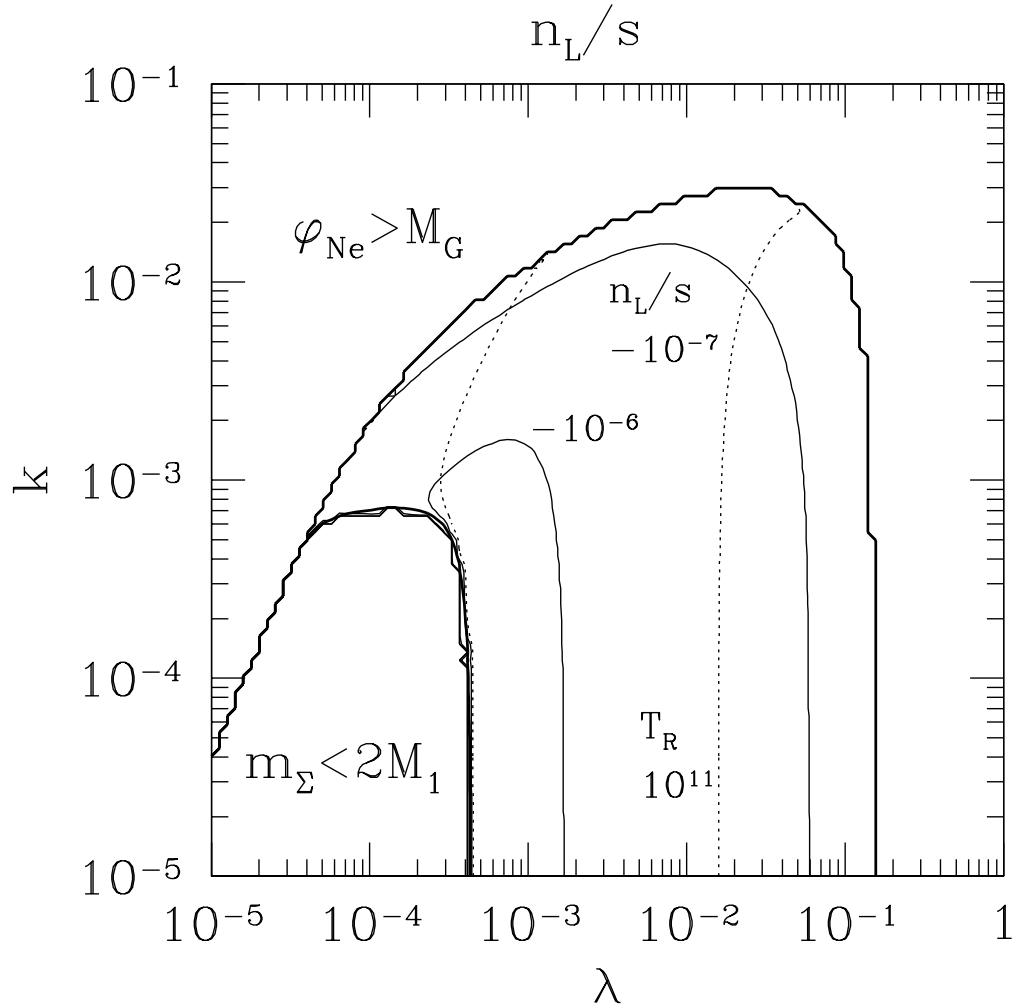

FIG. 6. The contour lines of the lepton asymmetry $n_{L} / s$ in the hybrid inflation model for the case $M_{1} \simeq 10^{12} \mathrm{GeV}(a+d=1)$. The contour lines are all shown by the thin solid lines and corresponding values of $n_{L} / s$ are also represented. We also show the contour lines of the reheating temperature by the dotted lines and corresponding values of $T_{R}$ are also represented in unit of $\mathrm{GeV}$. The upper bound on $k$ from the requirement $\varphi_{N_{e}}<M_{G}$ and the lower bound on $k$ from $m_{\varphi}=m_{\Sigma}>2 M_{1}$ are both shown by the thick solid lines. 


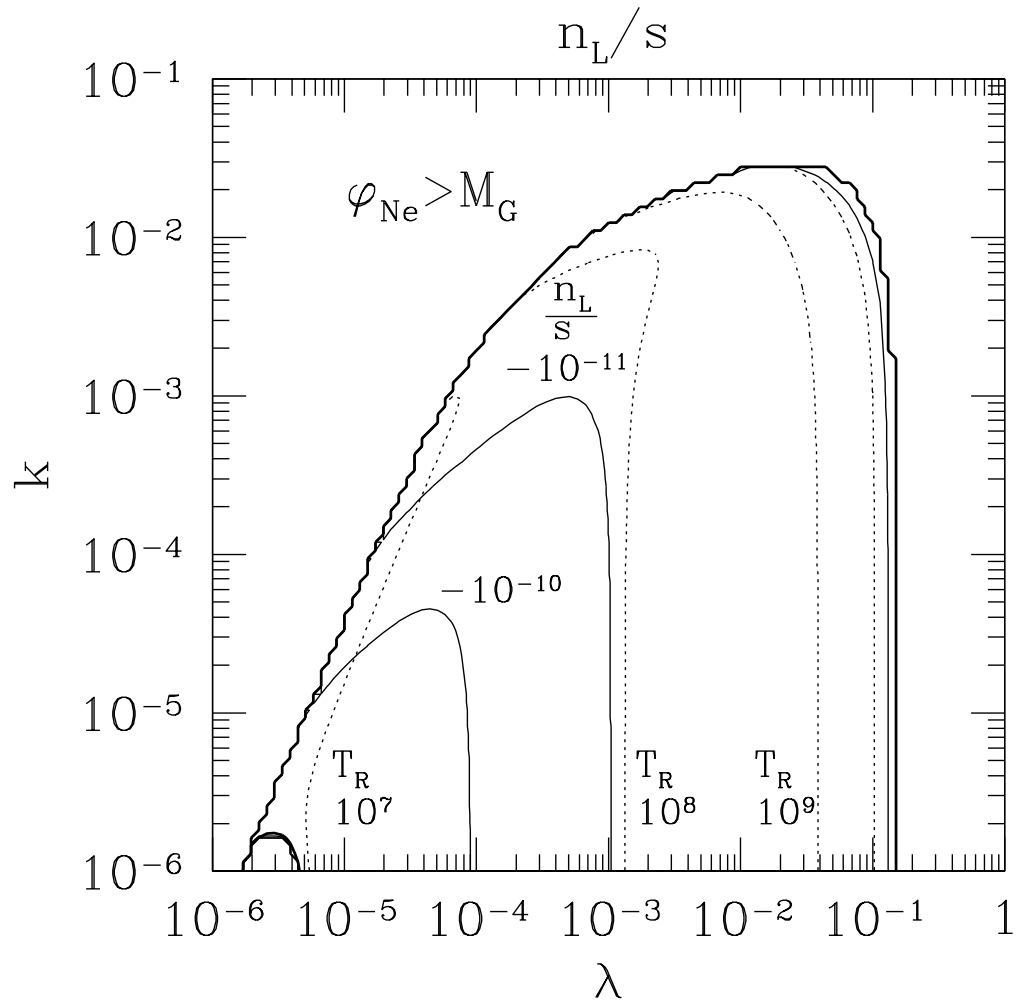

FIG. 7. The contour lines of the lepton asymmetry $n_{L} / s$ in the hybrid inflation model for the case $M_{1} \simeq 3 \times 10^{9} \mathrm{GeV}(a+d=2)$. The contour lines are all shown by the thin solid lines and corresponding values of $n_{L} / s$ are also represented. We also show the contour lines of the reheating temperature by the dotted lines and corresponding values of $T_{R}$ are also represented in unit of $\mathrm{GeV}$. The upper bound on $k$ from the requirement $\varphi_{N_{e}}<M_{G}$ and the lower bound on $k$ from $m_{\varphi}=m_{\Sigma}>2 M_{1}$ are both shown by the thick solid lines. 
We have, so far, identified the $U(1)$ gauge symmetry in the hybrid inflation model with the $B-L$ symmetry. We now consider the case where the $U(1)$ symmetry is nothing related to the $B-L$ symmetry and even completely decoupled from the SUSY standard-model sector. The role of the $U(1)$ gauge symmetry is only to eliminate an unwanted flat direction in Eq. (21).

In this case the $\Sigma$ field may decay through the nonrenormalizable interactions (33). On the other hand, the decay of the inflaton $\varphi$ is much suppressed due to the absence of the interaction (19). Thus, we introduce a new interaction in the Kähler potential as

$$
K=h \phi^{*} H_{u} H_{d}+\text { h.c. . }
$$

(A natural symmetry allowing the Kähler potential (41) will be discussed in the Appendix A.) Through this interaction the inflaton $\varphi$ may decay faster than the $\Sigma$ field for $h \simeq \mathcal{O}(1) \amalg^{\boxplus}$ and the reheating temperature $T_{R}$ is given by the decay of the $\Sigma$ field. Since the decay rate (34) is very small compared with Eq. (32), the reheating temperature $T_{R}$ becomes much lower than in the previous model. The inflation dynamics is almost the same as in the previous hybrid inflation. The difference of the results on various parameters, $\mu,\langle\Psi\rangle$ and $m_{\varphi}$ from those in the previous model may arise, in principle, from the different values of the

${ }^{14}$ Without the Kähler potential (41), the inflaton $\varphi$ decays through interactions in the Kähler potential $K \simeq\left|N_{1}\right|^{2}|\Psi|^{2}+\left|N_{1}\right|^{2}|\bar{\Psi}|^{2}$. The rate of this inflaton decay into two $\widetilde{N}_{1}$ is estimated as

$$
\Gamma\left(\varphi \rightarrow \widetilde{N_{1}} \widetilde{N_{1}}\right) \simeq \frac{1}{8 \pi}\langle\Psi\rangle^{2} M_{1}^{2} m_{\varphi}
$$

In this case the inflaton decay is slower than the decay of the $\Sigma$ field since $m_{\varphi}=m_{\Sigma}>2 M_{1}$ [see Eq. (34)], and the reheating temperature is determined by the decay rate (40). Then, the leptogenesis takes place in the inflaton decays into two $\widetilde{N_{1}}$. (Note that $B_{r} \simeq 1$ is ensured.) We find that the required lepton asymmetry is obtained with lower reheating temperature such as $T_{R} \simeq 10^{6} \mathrm{GeV}$ for the region $\lambda \simeq 10^{-4}-10^{-3}$ and $k \lesssim 10^{-2}$ (see Fig. 9).

${ }^{15}$ We find $\Gamma_{\varphi} \gtrsim \Gamma_{\Sigma}$ for $h^{2} \gtrsim C\langle\Psi\rangle^{2} \simeq 10^{-6}$. 


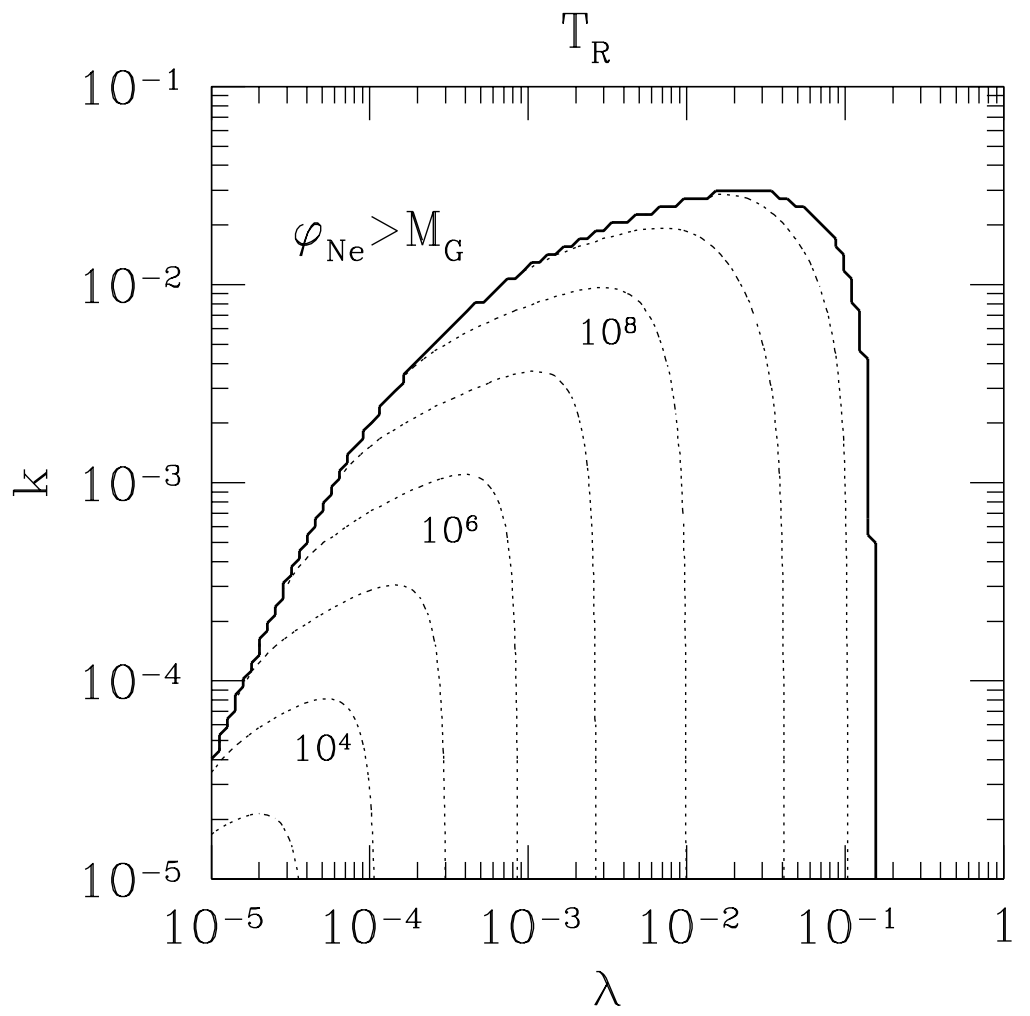

FIG. 8. The contour lines of the reheating temperature $T_{R}$ in the hybrid inflation model without the $B-L$ symmetry. The contour lines are all shown by the dotted lines and corresponding values of $T_{R}$ are also represented in unit of $\mathrm{GeV}$. The upper bound on $k$ from the requirement $\varphi_{N_{e}}<M_{G}$ is shown by the thick solid line.

reheating temperatures $T_{R}$ as shown in Fig. 8. However, we find no sizable difference in the obtained values of $\mu,\langle\Psi\rangle$ and $m_{\varphi}$ in between the present and the previous models.

We turn to estimate the lepton asymmetry in this hybrid inflation model. We find that too small lepton asymmetry is obtained for $M_{1} \simeq 3 \times 10^{9} \mathrm{GeV}(a+d=2)$ because of the small lepton-asymmetry parameter $\epsilon_{1}$ [see Eq. (12)], and hence we concentrate our discussion to the case of $M_{1} \simeq 10^{12} \mathrm{GeV}(a+d=1)$ in the following analysis. It is clear that the total decay rate (34) of the $\Sigma$ field is much smaller than the decay rate of $N_{1}$ in Eq. (8) and our approximation to derive Eq. (15) is justified. We show the $n_{L} / s$ in Fig. 9 assuming 
$B_{r}=1 . \square$ It is found that the required lepton asymmetry to account for the present baryon asymmetry can be generated in a wide parameter region $k \lesssim 10^{-2}$ and $\lambda \simeq 10^{-3}-10^{-2}$ with the reheating temperature of $T_{R} \simeq 10^{6}-10^{8} \mathrm{GeV}$. It is extremely interesting that we obtain the desired result, $n_{L} / s \simeq-10^{-10}$ and $T_{R} \simeq 10^{6} \mathrm{GeV}$, for $\lambda \simeq 10^{-3}$ and $k \lesssim 10^{-3}$. As shown in Ref. [12], we may avoid the overproduction of gravitinos in the full gravitino mass region of $m_{3 / 2} \simeq 100 \mathrm{GeV}-1 \mathrm{TeV}$ with such a low reheating temperature $T_{R} \simeq 10^{6} \mathrm{GeV}$. Notice that the gravitinos are also produced in the reheating process of the inflaton $\varphi$ decay. However, they are diluted by the entropy production of the $\Sigma$ decays and become negligible.

\footnotetext{
${ }^{16}$ In this model, the $\Sigma$ field decays not only into the heavy Majorana neutrinos $N_{1}$ but also into the SUSY standard-model particles through the nonrenormalizable interactions (33) and hence $B_{r} \simeq 1$ is not automatic.
} 


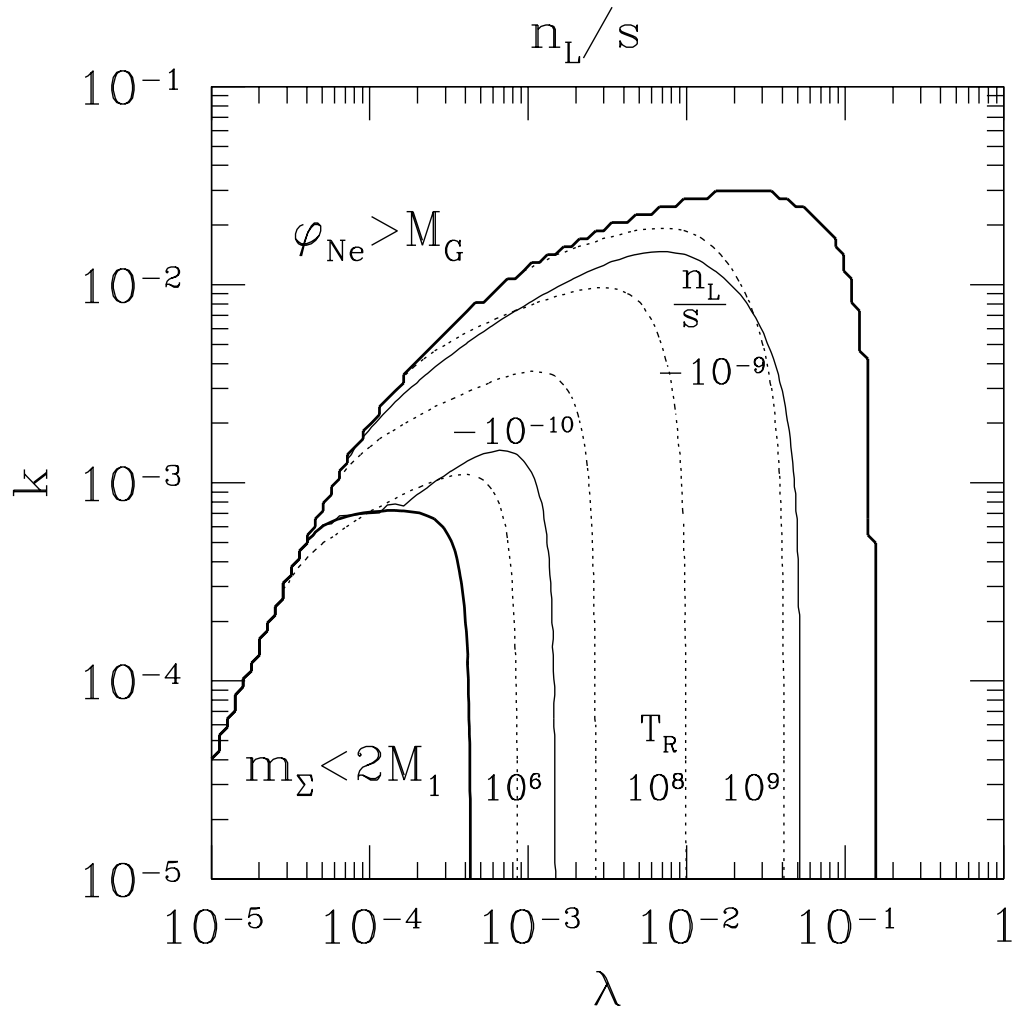

FIG. 9. The contour lines of the lepton asymmetry $n_{L} / s$ in the hybrid inflation model without the $B-L$ symmetry for the case $M_{1} \simeq 10^{12} \mathrm{GeV}(a+d=1)$. The contour lines are all shown by the thin solid lines and corresponding values of $n_{L} / s$ are also represented. Here we have assumed the branching ratio of $\Sigma \rightarrow N_{1} N_{1}$ decay channel $B_{r}=1$ for the estimation of $n_{L} / s$. We also show the contour lines of the reheating temperature by the dotted lines and corresponding values of $T_{R}$ are also represented in units of $\mathrm{GeV}$. The upper bound on $k$ from the requirement $\varphi_{N_{e}}<M_{G}$ and the lower bound on $k$ from $m_{\varphi}=m_{\Sigma}>2 M_{1}$ are both shown by the thick solid lines. 


\section{LEPTOGENESIS IN NEW INFLATION}

As seen in the previous section, the SUSY hybrid inflation with $T_{R} \simeq 10^{6}-10^{8} \mathrm{GeV}$ is successful to produce the sufficient lepton asymmetry to account for the baryon number in the present universe. However, we need small couplings, $k \lesssim 10^{-2}$ and $\lambda \lesssim 10^{-2}$ to obtain the low reheating temperature $T_{R} \simeq 10^{6}-10^{8} \mathrm{GeV}$. Although this is not a problem in the model, it is very important to find other inflation models which naturally produce the required low reheating temperature. The new inflation [28] is a well-known candidate for such inflation. Therefore, we investigate, in this section, whether the leptogenesis mechanism works in the new inflationary universe.

To perform a definite analysis we propose a SUSY new inflation model, $\square$ which has the following superpotential $W$ and Kähler potential $K$ for supermultiplets $\phi(x, \theta)$ and $\chi(x, \theta)$;

$$
\begin{aligned}
& W=\chi\left(v^{2}-g \phi^{n}\right) \\
& K=|\phi|^{2}+|\chi|^{2}+\frac{\kappa_{1}}{4}|\phi|^{4}+\kappa_{2}|\phi|^{2}|\chi|^{2}+\frac{\kappa_{3}}{4}|\chi|^{4} \cdots
\end{aligned}
$$

where $g, \kappa_{1}, \kappa_{2}$ and $\kappa_{3}$ are constants of order unity, $v$ the energy scale of the inflation, and the ellipsis denotes higher order terms. The superpotential (42) is naturally obtained, for example, by imposing $U(1)_{R} \times Z_{n}$ symmetry.

From Eqs. (22), (42) and (43) we find a SUSY vacuum, ${ }^{18}$

$$
\langle\phi\rangle=\left(\frac{v^{2}}{g}\right)^{\frac{1}{n}}, \quad\langle\chi\rangle=0 .
$$

The potential of scalar fields for $\phi, \chi \ll 1$ is given by

$$
V \simeq v^{4}-g v^{2}\left(\phi^{n}+\phi^{* n}\right)+g^{2}|\phi|^{2 n}+\left(1-\kappa_{2}\right) v^{4}|\phi|^{2}-\kappa_{3} v^{4}|\chi|^{2}
$$

\footnotetext{
17 Our discussion below can be also applied to the new inflation model proposed by Izawa and Yanagida [29], which offers a scalar potential for the inflaton field similar to Eq. 46).

18 We always take $g$ and $v^{2}$ real and positive by using the phase rotations of $\chi$ and $\phi$.
} 
where we have also used the same symbols $\phi$ and $\chi$ for the scalar components of corresponding supermultiplets and neglected the higher order terms represented by the ellipsis in Eq. (43).

For $g>0$ and $k \equiv \kappa_{2}-1>0$, we can identify the inflaton field $\varphi$ with the real part of the scalar field $\phi$. Moreover, if $\kappa_{3}<-\frac{3}{4}, \chi$ receives a mass which is larger than $(3 / 2) H_{I}$ with the Hubble parameter $H_{I}$ during the new inflation, and hence $\chi$ settles down at $\chi=0$ quickly. Hereafter, we assume $\kappa_{3}<-\frac{3}{4}$ and set $\chi=0$. Then, the inflaton potential near the origin $(\varphi \simeq 0)$ is obtained as

$$
V(\varphi) \simeq v^{4}-\frac{k}{2} v^{4} \varphi^{2}-\frac{g}{2^{\frac{n}{2}-1}} v^{2} \varphi^{n}
$$

The slow-roll conditions for the inflation Eqs. (28) and (29) are satisfied when

$$
\begin{aligned}
& 0<k \lesssim 1, \\
& 0<\varphi \lesssim \varphi_{f} \equiv \sqrt{2}\left[\frac{(1-k) v^{2}}{g n(n-1)}\right]^{\frac{1}{n-2}} .
\end{aligned}
$$

Then, the new inflation takes place when the inflaton $\varphi$ rolls down along the potential Eq. (46) from $\varphi \simeq 0$ to $\varphi_{f}$. 19

In the true vacuum Eq. (44), the inflaton $\varphi$ has a mass as

$$
m_{\varphi} \simeq n g^{\frac{1}{n}} v^{2-\frac{2}{n}}
$$

We assume that the inflaton decays through nonrenormalizable interactions in the Kähler potential such asto

\footnotetext{
19 The initial value of the inflaton field $\varphi$ should be taken near the local maximum of the potential $\varphi \simeq 0$ to obtain a successful new inflation [30]. There seems no reason to take such an initial value, since the potential should be flat enough for $\varphi$ to roll down slowly. However, there have been found some dynamical mechanisms [31,32] to solve this initial value problem.

20 Suitable charge assignment of $Z_{n}$ symmetry for the Higgs supermultiplets $H_{u}$ and $H_{d}$ forbids a Kähler potential $K=\phi^{*} H_{u} H_{d}$.
} 


$$
K=\sum_{i} \lambda_{i}|\phi|^{2}\left|\psi_{i}\right|^{2}
$$

where $\psi_{i}$ denote supermultiplets for SUSY standard-model particles including the heavy Majorana neutrinos, and $\lambda_{i}$ are coupling constants of order unity. Then, the decay rate $\Gamma_{\varphi}$ is estimated as

$$
\Gamma_{\varphi} \simeq \frac{1}{8 \pi} \sum_{i} \lambda_{i}^{2}\langle\phi\rangle^{2} m_{\varphi}^{3}
$$

which leads to

$$
T_{R} \simeq 0.092 C^{\prime}\langle\phi\rangle m_{\varphi}^{\frac{3}{2}}
$$

where $C^{\prime} \equiv \sqrt{\sum_{i}\left|\lambda_{i}\right|^{2}}$ and we take $C^{\prime}=1$ in the following discussion. Then, the scale $v$ of the new inflation in Eq. (42) is determined from the $e$-fold number $N_{e}$ of the present horizon, the amplitude and the spectrum index $n_{s}$ of the primordial density fluctuations $\delta \rho / \rho$.

The number of $e$-foldings $N_{e}$ is given by Eq. (37). The present new inflation gives

$$
\begin{aligned}
N_{e} & =\int_{\varphi_{f}}^{\varphi_{N_{e}}} d \varphi \frac{V(\varphi)}{V^{\prime}(\varphi)} \\
& \simeq \int_{\widetilde{\varphi}}^{\varphi_{N_{e}}} d \varphi \frac{v^{4}}{-k v^{4} \varphi}+\int_{\varphi_{f}}^{\widetilde{\varphi}} d \varphi \frac{v^{4}}{-\left(n g / 2^{\frac{n}{2}-1}\right) v^{2} \varphi^{n-1}},
\end{aligned}
$$

where

$$
\widetilde{\varphi}=\sqrt{2}\left(\frac{k v^{2}}{n g}\right)^{\frac{1}{n-2}}
$$

and $\varphi_{N_{e}}$ is the value of the field $\varphi$ when the present universe crossed the horizon. Here we have assumed that $\varphi_{N_{e}}<\tilde{\varphi}$. $\square$ Then, we find

$$
\varphi_{N_{e}} \simeq \sqrt{2}\left(\frac{k v^{2}}{n g}\right)^{\frac{1}{n-2}} \exp \left[-k\left(N_{e}+\frac{n k-1}{(n-2) k(1-k)}\right)\right] \text {. }
$$

\footnotetext{
21 This condition corresponds to $k \gtrsim 1 /\left[N_{e}(n-2)\right]$.
} 
The amplitude of the primordial density fluctuations $\delta \rho / \rho$ due to the inflation should be normalized by the data on anisotropies of the CMBR observed by the COBE experiments as in the hybrid inflation. From Eq. (39) we find

$$
\frac{v^{2}}{k \varphi_{N_{e}}} \simeq 5.3 \times 10^{-4} \text {. }
$$

Furthermore, the COBE observations show the spectrum index $n_{s}$ as $n_{s}=1.0 \pm 0.2$ [27] 22 In the present new inflation model the index $n_{s}$ is calculated as 29

$$
n_{s} \simeq 1-2 k
$$

Therefore, we take $0.01 \lesssim k \lesssim 0.1$ in the following analysis assuming the coupling $k$ not extremely small.23

We calculate the scale $v$ for given $n, g$ and $k$, which are shown in Fig. 10. The vev $\langle\phi\rangle$, the inflaton mass $m_{\varphi}$ and the reheating temperature $T_{R}$ are found in Figs. 11, 12 and 13, respectively. From Fig.13 we find that the new inflation model with the power index $n=4$, 5 and 6 naturally offers the low reheating temperature $T_{R} \lesssim 10^{8} \mathrm{GeV}$. For the cases $n=7$ and 8, we obtain $T_{R} \lesssim 10^{8} \mathrm{GeV}$ for the region $k \simeq 0.05-0.1$ and $0.07-0.1$, respectively.

\footnotetext{
${ }^{22}$ For the case of the scale invariant spectrum we have $n_{s}=1$.

${ }^{23}$ Notice that our assumption $\left(\varphi_{N_{e}}<\widetilde{\varphi}\right.$ ) in deriving Eq. (53) is justified (see the footnote 21).
} 


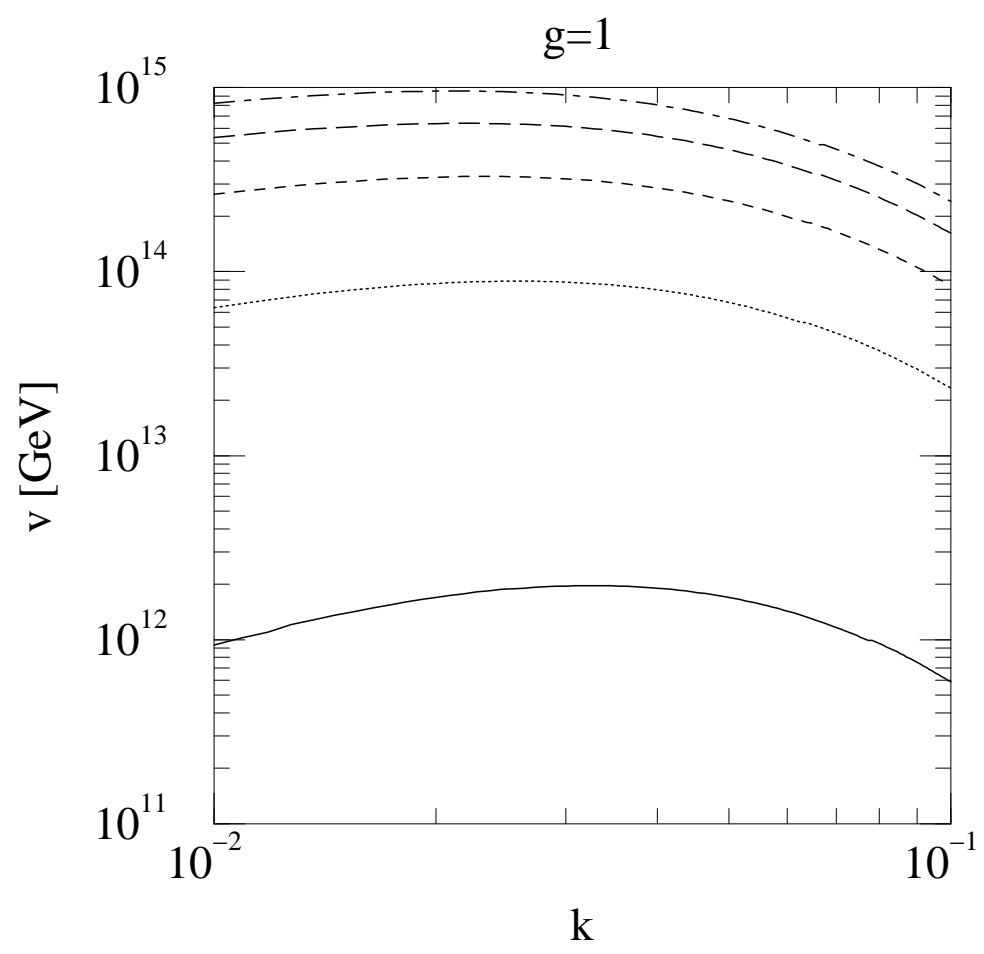

FIG. 10. The scale $v$ of the new inflation for $g=1$. We take the index $n$ as $n=4,5,6,7$ and 8 from the bottom to the top.

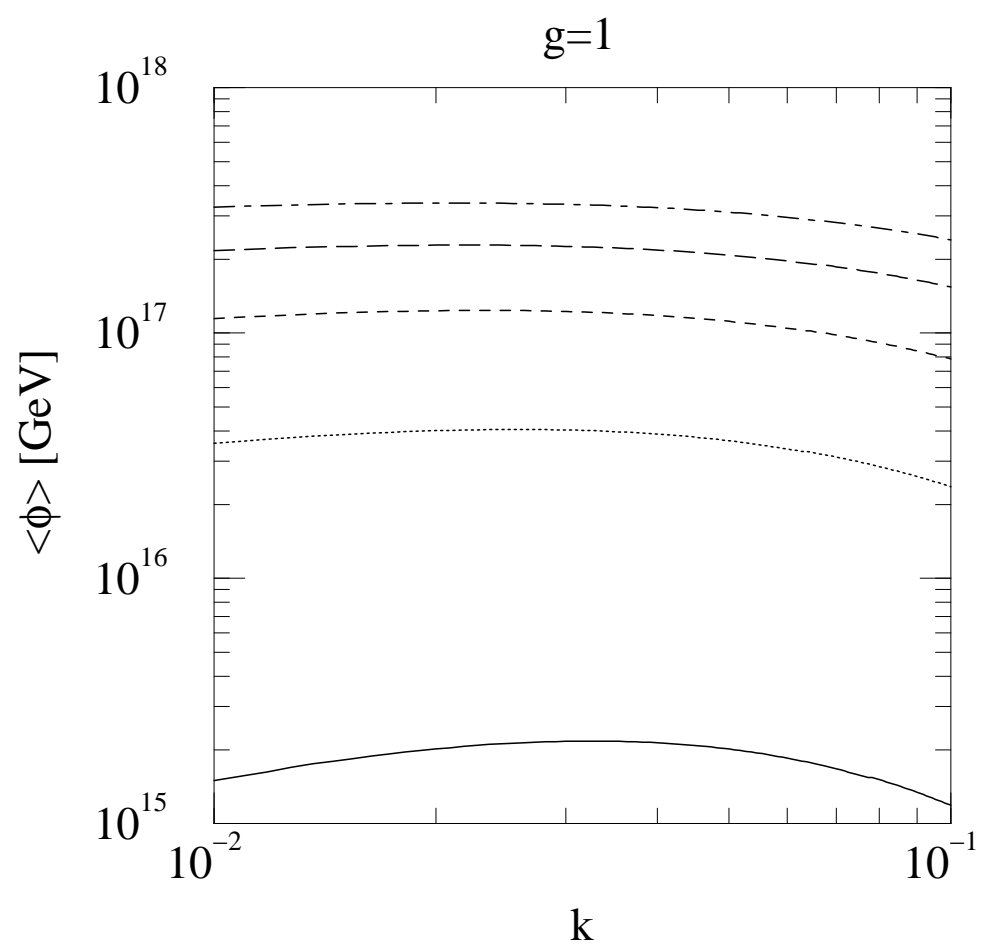

FIG. 11. The vev $\langle\phi\rangle$ of the new inflation for $g=1$. We take the index $n$ as $n=4,5,6,7$ and 8 from the bottom to the top. 


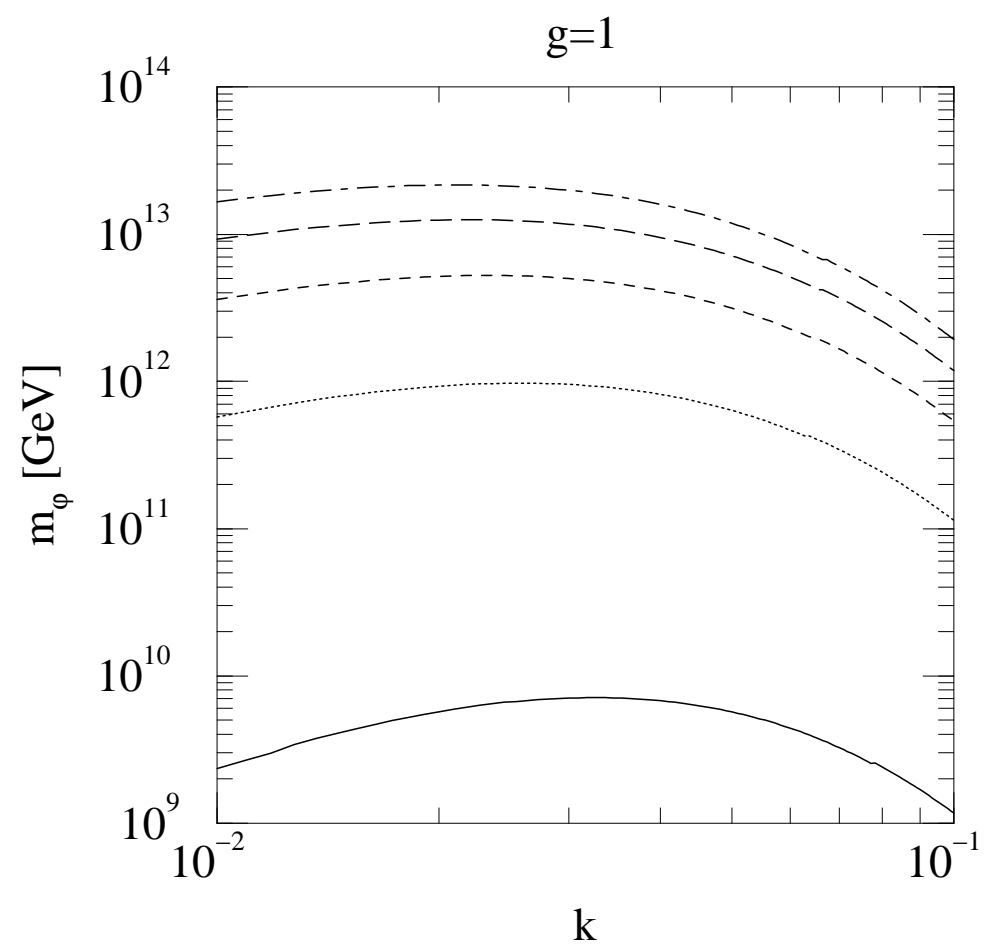

FIG. 12. The inflation mass $m_{\phi}$ of the new inflation for $g=1$. We take the index $n$ as $n=4$, 5, 6, 7 and 8 from the bottom to the top.

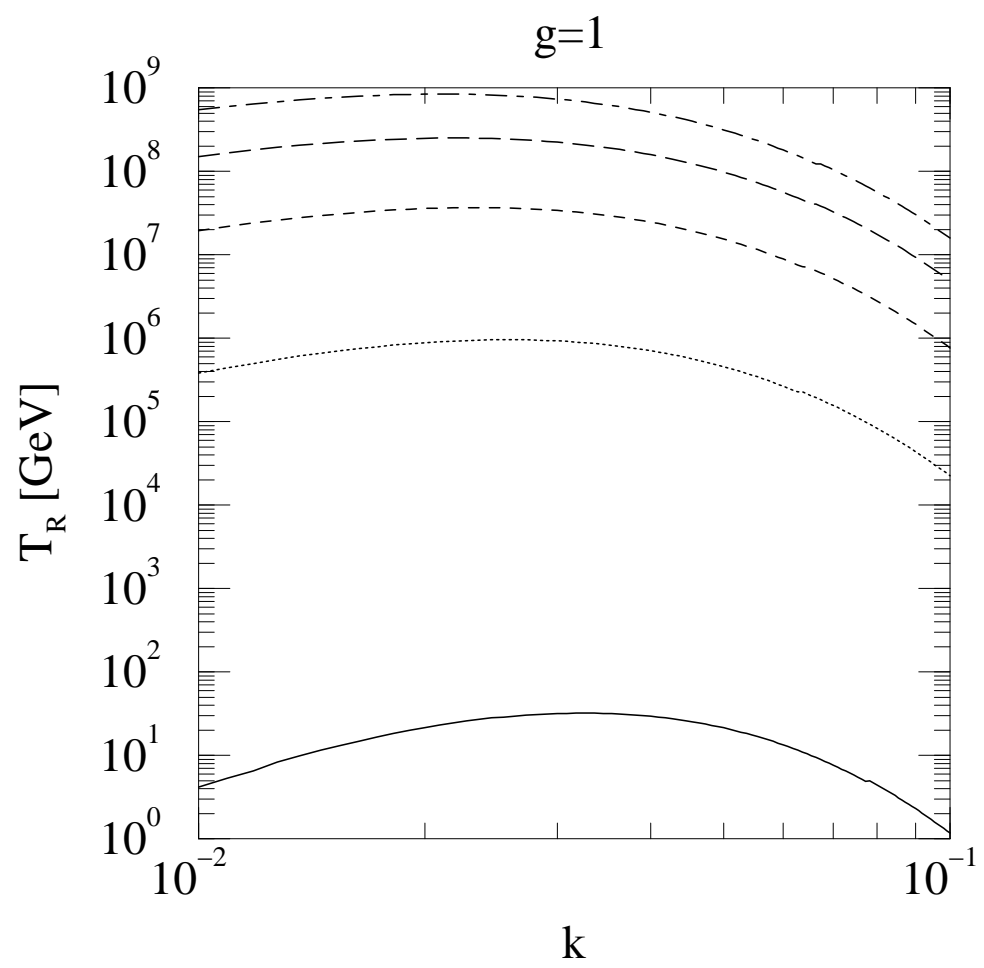

FIG. 13. The reheating temperature $T_{R}$ of the new inflation for $g=1$. We take the index $n$ as $n=4,5,6,7$ and 8 from the bottom to the top. 


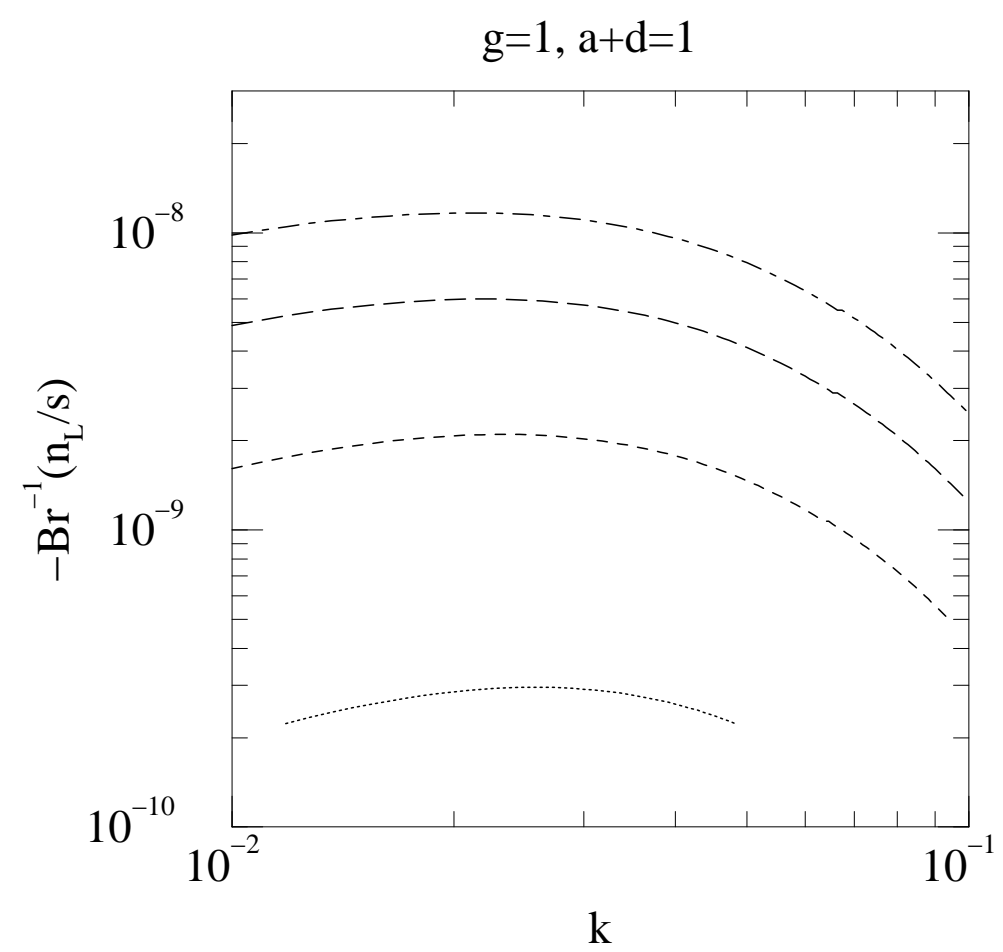

FIG. 14. The lepton asymmetry produced via the decays of $N_{1}$ in the new inflation. The index $n$ is taken as $n=5,6,7$ and 8 from the bottom to the top. We take the FN charges $a$ and $d$ as $a+d=1$. For $n=5$, the regions $k<1.2 \times 10^{-2}$ and $k>4.8 \times 10^{-2}$ are excluded since $m_{\varphi} \leq 2 M_{1}$

We turn to discuss the leptogenesis in this new inflation model. Here we also consider only the leptogenesis via the decays of the lightest Majorana neutrinos $N_{1}$ produced in the inflaton decay. As well as in the previous hybrid inflation, the inflaton decay rate $\Gamma_{\varphi} \simeq T_{R}^{2}$ is much smaller than the decay rate of $N_{1}$ in Eq. (8), and the decays of $N_{1}$ occur just after produced in the inflaton decay. Thus, the ratio of the lepton-number density $n_{L}$ to the entropy density $s$ is also given by Eq. (15). Notice that the new inflation model with $n=4$ gives so low reheating temperature as $T_{R} \simeq 1 \mathrm{GeV}-10 \mathrm{GeV}$ (see Fig. 13), that the required amount of lepton asymmetry can not be generated [see Eq. (15)], and hence we discard this case.

For the case $n=5,6,7$ and 8, we show the produced lepton asymmetry in Fig. 14 and Fig. 15 by taking $a+d=1$ and $a+d=2$, respectively. Here we have taken a smaller value of $M_{1}$ as $M_{1} \simeq \epsilon^{2(a+d)} 10^{14} \mathrm{GeV}$ [see Eq. (7)] to obtain a wider allowed region, taking account of $\mathcal{O}(1)$ ambiguities in the FN model. 


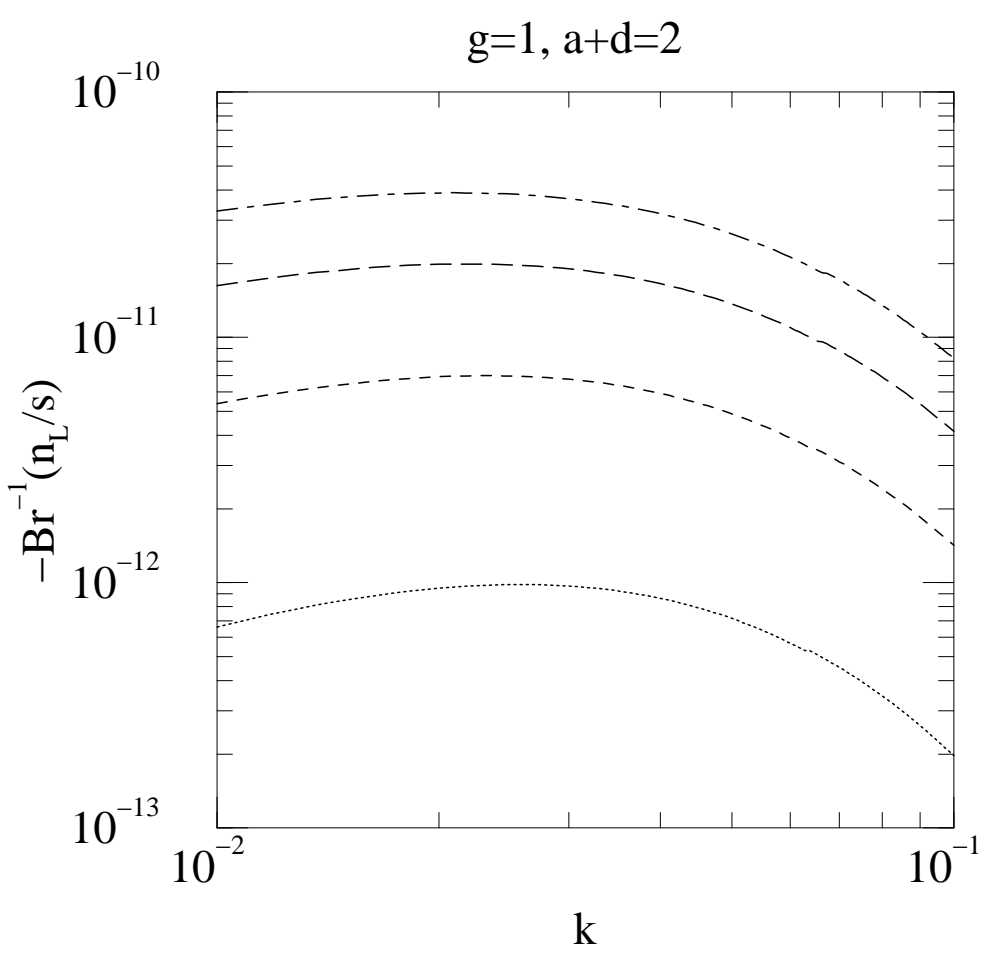

FIG. 15. The lepton asymmetry produced via the decays of $N_{1}$ in the new inflation. The index $n$ is taken as $n=5,6,7$ and 8 from the bottom to the top. We take the FN charges $a$ and $d$ as $a+d=2$.

First, we consider the case $a+d=1$ and $M_{1} \simeq 3 \times 10^{11} \mathrm{GeV}$. In order to allow the decay $\varphi \rightarrow N_{1} N_{1}$ the inflaton mass should be larger than $2 M_{1}$, which excludes the regions $k<1.2 \times 10^{-2}$ and $k>4.8 \times 10^{-2}$ for the case $n=5$. We find from Fig. 14 that the sufficient lepton asymmetry can be generated for $n=5,6,7$ and 8 with the low enough reheating temperature of $T_{R} \simeq 10^{6}-10^{8} \mathrm{GeV}$ (see Fig. 13). It should be noted that the required lepton asymmetry $n_{L} / s \simeq-10^{-10}$ is obtained for $T_{R} \simeq 10^{6} \mathrm{GeV}$ when $B_{r} \simeq 1$ and $n=5$, and we can avoid the overproduction of gravitinos even if $m_{3 / 2} \simeq 100 \mathrm{GeV}-500 \mathrm{GeV}$. This is a crucial result in the present analysis. On the other hand, when $M_{1} \simeq 10^{9} \mathrm{GeV}$ (i.e., $a+d=2$ ), we find from Fig. 15 that the leptogenesis does not work well."4

\footnotetext{
${ }^{24}$ For the case $\mathrm{n}=8$, we obtain $B_{r}^{-1}\left(n_{L} / s\right) \simeq-3 \times 10^{-11}$ in the region $10^{-2} \lesssim k \lesssim 0.4$. However, the reheating temperature is $T_{R} \gtrsim 10^{8} \mathrm{GeV}$ and hence the cosmological gravitino problem is still
} 


\section{LEPTOGENESIS IN TOPOLOGICAL INFLATION}

Finally, we discuss the leptogenesis in a topological inflation [15]. If the vev of the inflaton exceeds the gravitational scale, the inflaton potential (46) in the new inflation model is nothing but the inflaton potential for the topological inflation [33].

For example, we consider the SUSY topological inflation model proposed in Ref. [34]. The superpotential and Kähler potential in the model are given by

$$
\begin{aligned}
& W=v^{2} \chi\left(1-g \phi^{2}\right), \\
& K=|\chi|^{2}+|\phi|^{2}+\frac{\kappa_{1}}{4}|\phi|^{4}+\kappa_{2}|\chi|^{2}|\phi|^{2}+\frac{\kappa_{3}}{4}|\chi|^{4}+\cdots,
\end{aligned}
$$

where $v$ is the energy scale of the inflation, $g, \kappa_{1}, \kappa_{2}$ and $\kappa_{3}$ coupling constants of order unity. These potentials possess $U(1)_{R} \times Z_{2}$ symmetry, and the $U(1)_{R}$ charge of $\phi(\chi)$ is $-2(0)$ and $\phi(\chi)$ is odd (even) under the $Z_{2}$. This discrete symmetry is crucial for the topological inflation.

From the scalar potential (22), and Eqs. (58) and (59) we find a SUSY-invariant vacuum,

$$
\langle\chi\rangle=0, \quad\langle\phi\rangle=\frac{1}{\sqrt{g}},
$$

in which the potential energy vanishes. The potential for the region $|\chi|,|\phi| \ll 1$ is written approximately as

$$
V \simeq v^{4}\left|1-g \phi^{2}\right|^{2}+\left(1-\kappa_{2}\right) v^{4}|\phi|^{2}-\kappa_{3} v^{4}|\chi|^{2} .
$$

The scalar components of the supermultiplets are denoted by the same letters as the corresponding supermultiplets. Hereafter, we set $\chi=0$ assuming $\kappa_{3}<-3 / 4$ as in the new inflation. For $g>0$ and $\kappa_{2}<1$, the inflaton field $\varphi$ is identified with the real part of $\phi$ and the potential around the origin is given by

$$
V(\varphi) \simeq v^{4}-\frac{k}{2} v^{4} \varphi^{2}
$$

unsolved in this region. 


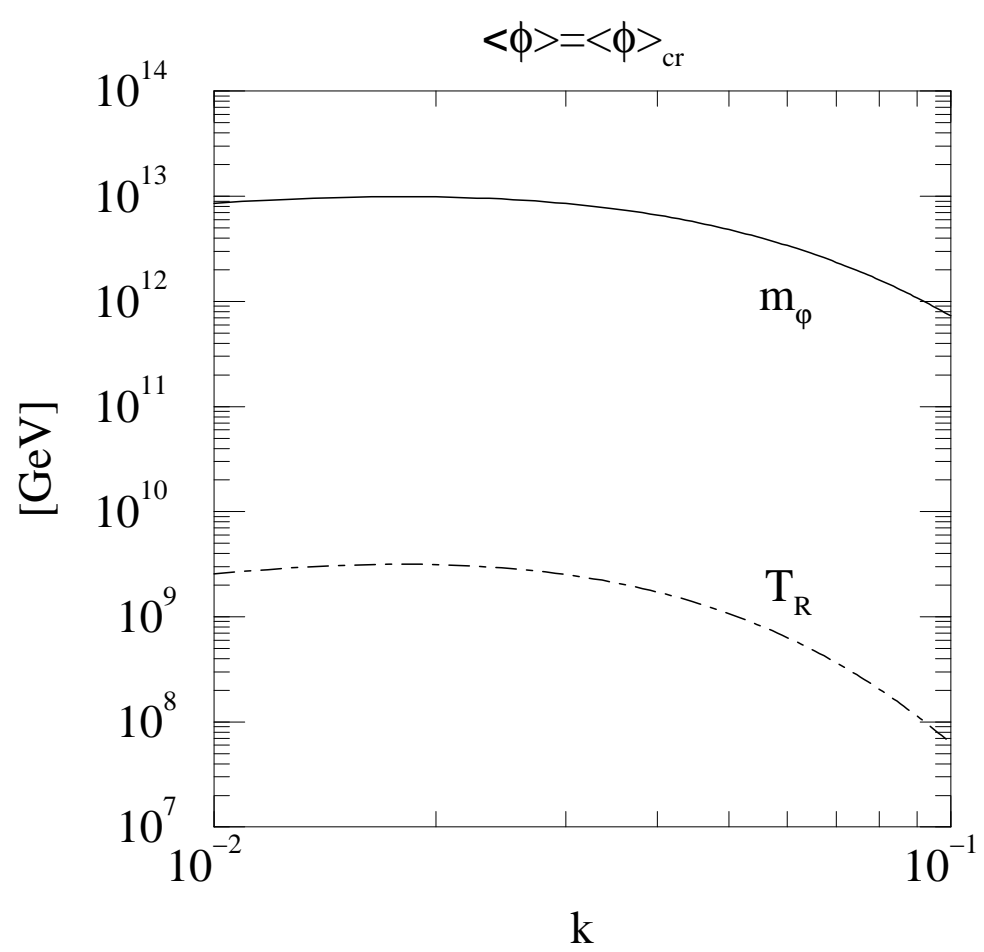

FIG. 16. The inflaton mass $m_{\varphi}$ and the reheating temperature $T_{R}$ of the topological inflation model. We take $\langle\phi\rangle=\langle\phi\rangle_{c r} \simeq 1.7 M_{G}$. The solid and dot-dashed lines represent $m_{\varphi}$ and $T_{R}$, respectively.

where $k \equiv 2 g+\kappa_{2}-1.2$ In the true vacuum (60) the inflaton mass is estimated as

$$
m_{\varphi} \simeq 2 \sqrt{g} v^{2}=\frac{2 v^{2}}{\langle\phi\rangle}
$$

A topological inflation takes place if the vev of $\phi$ is of order of the gravitational scale $M_{G}$ [33]. As verified in Ref. [35], it should be

$$
\langle\phi\rangle \geq\langle\phi\rangle_{c r} \simeq 1.7
$$

The slow-roll conditions Eqs. (28) and (29) are satisfied when $0<k<1$ and $\varphi \lesssim \varphi_{f}$ where $\varphi_{f}$ is expected to be of order of the gravitational scale and we take $\varphi_{f}=1$.

Then, the scale of this topological inflation is determined by the $e$-fold number $N_{e}$, the density fluctuations $\delta \rho / \rho$ and the spectrum index $n_{s}$. The $e$-fold number can be written as

\footnotetext{
25 This is nothing but the potential (46) in the new inflation model with $n=2$.
} 


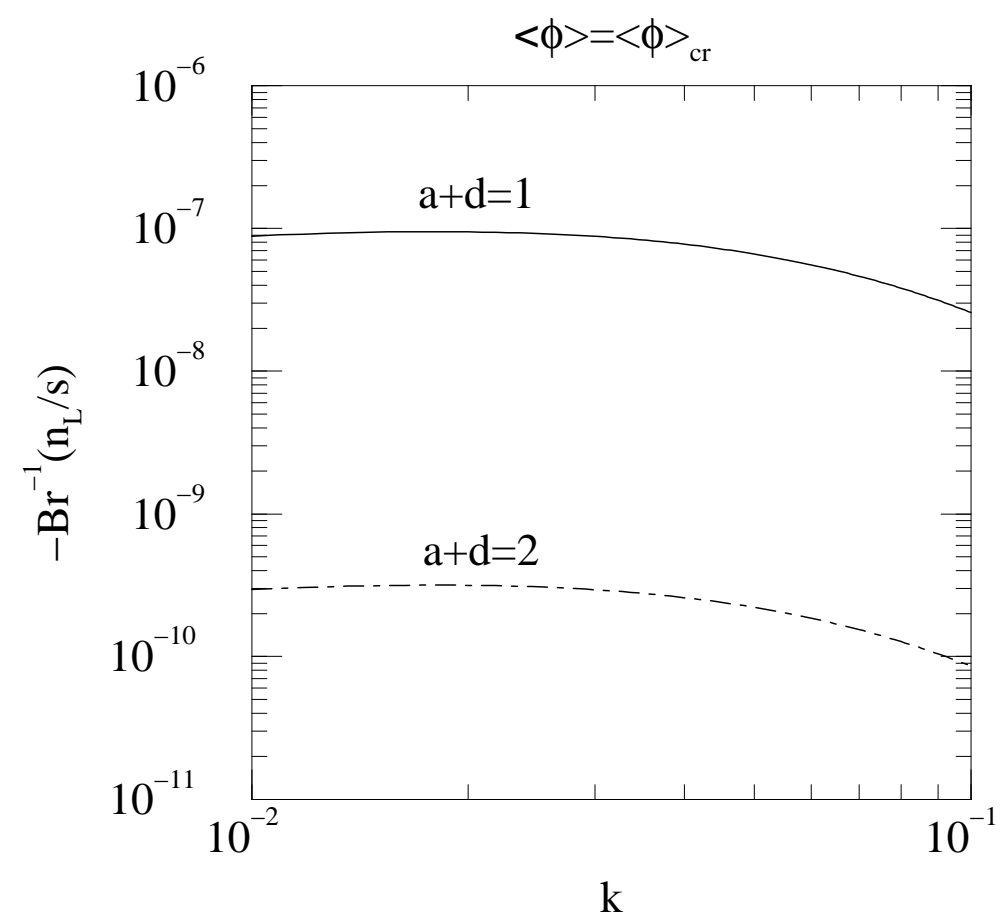

FIG. 17. The lepton asymmetries, $n_{L} / s$, in the topological inflation model for the case $a+d=1$ (the solid line) and $a+d=2$ (the dot-dashed line). We take $\langle\phi\rangle=\langle\phi\rangle_{c r} \simeq 1.7 M_{G}$.

$$
N_{e} \simeq \int_{\varphi_{f}}^{\varphi_{N_{e}}} d \varphi \frac{V}{V^{\prime}} \simeq \frac{1}{k} \ln \left(\frac{\varphi_{f}}{\varphi_{N_{e}}}\right)
$$

We also assume that the inflaton $\varphi$ decays through the interactions in the Kähler potential as shown in Eq. (50) and hence the reheating temperature of the topological inflation is also given by Eq. (52). $\delta \rho / \rho$ in the topological inflation can be calculated as

$$
\frac{\delta \rho}{\rho} \simeq \frac{1}{5 \sqrt{3} \pi} \frac{v^{2}}{k \varphi_{N_{e}}},
$$

which should be normalized by the COBE data in Eq. (56). Furthermore, the spectrum index $n_{s}$ is given by $n_{s} \simeq 1-2 k$, and hence we take $0.01 \lesssim k \lesssim 0.1$ as in the new inflation model. In Fig. 16 we show the inflation mass $m_{\varphi}$ and the reheating temperature $T_{R}$ of the topological inflation by taking $\langle\phi\rangle=\langle\phi\rangle_{c r} \simeq 1.7$. We see relatively high reheating temperatures compared with those in the new inflation. This is due to a larger value of $\langle\phi\rangle$.

Since the inflaton mass lies in the region $m_{\varphi} \simeq 10^{12}-10^{13} \mathrm{GeV}$ and then the inflaton always decays into two heavy Majorana neutrinos $N_{1}$ for the both $a+d=1$ and 2 cases. Here we also take the mass of the $N_{1}$ as $M_{1} \simeq \epsilon^{2(a+d)} 10^{14} \mathrm{GeV}$ [see Eq. (7)]. The inflaton 
decay rate is much smaller than the decay rate of $N_{1}$ in Eq. (8) and the estimation of the lepton asymmetry in Eq. (15) is also justified. The ratio of the lepton number to the entropy density, $n_{L} / s$, in the topological inflation is found in Fig. 17. For the both $a+d=1$ and 2 cases, we obtain lepton asymmetry $\left|n_{L} / s\right| \gtrsim 10^{-10}$ for $B_{r} \simeq 1$. However, if one requires the low reheating temperature in order to suppress sufficiently the gravitino abundance as $T_{R} \lesssim 10^{8} \mathrm{GeV}$, the coupling $k$ should be $k \gtrsim 0.092$. (The lowest reheating temperature $T_{R}=6.3 \times 10^{7} \mathrm{GeV}$ is obtained for $k=0.1$.) For $k \simeq 0.1$, the spectrum index $n_{s}$ deviates from the scale invariant one as $n_{s} \simeq 0.8$, and it will be testable in future satellite experiments [36] on anisotropies of the CMBR.

We should note finally that we may accommodate a late-time entropy production of order $10^{2}-10^{3}$ in the case of $a+d=1$, since the produced lepton asymmetry is large as $n_{L} / s \simeq-10^{-7}$ as shown in Fig. 17. If it is the case, the energy density of the gravitinos is diluted by the factor $10^{2}-10^{3}$, which allows the region of $T_{R} \simeq 10^{9} \mathrm{GeV}$ in Fig. 16.

\section{DISCUSSION AND CONCLUSIONS}

We have discussed, in this paper, the leptogenesis via the decays of the heavy Majorana neutrinos $N_{1}$ produced non-thermally in the inflaton decay. We have performed a detailed analysis on the leptogenesis taking three types of SUSY models for hybrid, new and topological inflations, and found that all of them are successful to produce the lepton-number asymmetry enough to account for the baryon asymmetry in the present universe. Here we have imposed the reheating temperatures $T_{R} \lesssim 10^{8} \mathrm{GeV}$ to suppress sufficiently the density of gravitinos for keeping the success of the BBN for $m_{3 / 2} \simeq \mathcal{O}(1) \mathrm{TeV}$. However, much lower reheating temperature such as $T_{R} \simeq 10^{6} \mathrm{GeV}$ is required 12 for $m_{3 / 2} \simeq 100 \mathrm{GeV}-500 \mathrm{GeV}$, which is only achieved in the hybrid or new inflation model. In other words, the leptogenesis is fully consistent with the BBN in a wide range of the gravitino mass of $m_{3 / 2} \simeq 100 \mathrm{GeV}-1$ $\mathrm{TeV}$ in the hybrid or new inflationary universe. It should be noted that the leptogenesis in these inflation models are also consistent with a class of the gauge-mediated SUSY breaking 
models [37], because the overclosure problem of the stable gravitino of $m_{3 / 2} \simeq 10 \mathrm{MeV}-1$ $\mathrm{GeV}$ can be avoided when the reheating temperature is $T_{R} \simeq 10^{6} \mathrm{GeV} 38$.

The above hybrid inflation with $T_{R} \simeq 10^{6} \mathrm{GeV}$ is realized by taking small couplings $k \lesssim 10^{-3}$ and $\lambda \simeq 10^{-3}$ (see Fig. 9), which predicts the breaking scale of the $U(1)$ gauge symmetry at $\langle\Psi\rangle=\langle\bar{\Psi}\rangle \simeq(4-6) \times 10^{15} \mathrm{GeV}$. This $U(1)$ breaking produces cosmic strings in the early universe. The cosmic strings with such a high breaking scale of $10^{15}-10^{16}$ $\mathrm{GeV}$ are very interesting, since they give additional contributions to the anisotropies of the CMBR [39], which may be testable in future satellite experiments [36]. We should note that the scale-invariant spectrum index $\left(n_{s} \simeq 1\right)$ is also predicted in the hybrid inflation model.

The successful new inflation with $T_{R} \simeq 10^{6} \mathrm{GeV}$ is given by taking the $k \simeq 0.01-0.05$ (see Figs. 13 and 14), which yields the spectrum index $n_{s} \simeq 0.98-0.90$. We expect that some parameter region of $n_{s}$ may be excluded (or confirmed) in the future experiments [36].

In the present paper, we have assumed the FN model for mass matrices of quarks and leptons. However, it is straightforward task to apply the present analysis to another model for Yukawa couplings for quarks and leptons to evaluate the asymmetry parameter $\epsilon_{1}$. The leptogenesis with more generic Yukawa matrices will be discussed in a future publication [40].

\section{ACKNOWLEDGEMENTS}

The authors would like to thank to Toshiyuki Kanazawa and Masahide Yamaguchi for useful discussion. This work was partially supported by the Japan Society for the Promotion

of Science (TA,KH) and "Priority Area: Supersymmetry and Unified Theory of Elementary Particles $(\sharp 707) ”(\mathrm{MK}, \mathrm{TY})$. 


\begin{tabular}{|c|ccc|cc|ccc|}
\hline \hline & $\phi$ & $\Psi$ & $\bar{\Psi}$ & $H_{u}$ & $H_{d}$ & $l_{i}$ & $e_{i}^{c}$ & $N_{i}$ \\
\hline$Q_{R}$ & 2 & 0 & 0 & $2-2 a$ & $3-3 a$ & $2 a-1$ & $a$ & 1 \\
\hline \hline
\end{tabular}

TABLE II. The $U(1)_{R}$ charges of various supermultiplets.

\section{APPENDIX A:}

In this appendix, we show a symmetry which naturally provides the superpotential (21) as well as the Kähler potential (41) for the inflation supermultiplet $\phi(x, \theta)$. It is very crucial to suppress $W=\lambda^{\prime} \phi H_{u} H_{d}$ in the hybrid inflation model.

We adopt $U(1)_{R} R$-symmetry and $R$-charges of relevant supermultiplets are found in Table [1]. We see that the dangerous superpotential $W=\lambda^{\prime} \phi H_{u} H_{d}$ is forbidden by choosing $a \neq 1$.

Now we discuss, by taking $a=0$ for example, a breaking of $U(1)_{R}$ to induce a Kähler potential (41), which is very important to cause the inflaton $\varphi$ decay in the second hybrid inflation model as explained in the text. We assume that the $U(1)_{R}$ symmetry is broken down to $Z_{3}$-symmetry by the vev of the $\Xi$ supermultiplet carrying a $U(1)_{R^{-c h a r g e}}+3$. Because of the positive $R$-charge of $\Xi$ the superpotential does not receive any $U(1)_{R^{-}}$breaking effects. However, we may have the following Kähler potential:

$$
K=f \Xi^{*} \phi^{*} H_{u} H_{d}+\text { h.c. . }
$$

Then, the non-vanishing vev of the $\Xi$ field gives rise to Eq. (41) and the coupling constant $h$ in Eq. (41) is given by

$$
h=f\left\langle\Xi^{*}\right\rangle
$$




\section{REFERENCES}

[1] G. 't Hooft, Phys. Rev. Lett. 37 (1976) 8, Phys. Rev. D14 (1976) 3432.

[2] V.A. Kuzmin, V.A. Rubakov, and M.E. Shaposhnikov, Phys. Lett. B155 (1985) 36.

[3] M. Fukugita and T. Yanagida, Phys. Rev. D42 (1990) 1285.

[4] I. Affleck and M. Dine, Nucl. Phys. B249 (1985) 361.

[5] See, for example, a recent review, J.M. Cline, hep-ph/9902328, and reference therein.

[6] T. Yanagida, in Proc. Workshop on the unified theory and the baryon number in the universe, (Tsukuba, 1979), eds. O. Sawada and S. Sugamoto, Report KEK-79-18 (1979); M. Gell-Mann, P. Ramond and R. Slansky, in "Supergravity" (North-Holland, Amsterdam, 1979) eds. D.Z. Freedman and P. van Nieuwenhuizen.

[7] Y. Fukuda et al. [Superkamiokande Collaboration], Phys. Lett. B433 (1998) 9; Phys. Lett. B436 (1998) 33; Phys. Rev. Lett. 81 (1998) 1562.

[8] M. Fukugita and T. Yanagida, Phys. Lett. B174 (1986) 45.

[9] See, for a recent review, W. Buchmüller and M. Plümacher, hep-ph/9904310; M. Plümacher, Nucl. Phys. B530 (1998) 207 and reference therein.

[10] K. Kumekawa, T. Moroi and T. Yanagida, Prog. Theor. Phys. 92 (1994) 437; G. Lazarides, hep-ph/9904428 and reference therein; G.F. Giudice, M. Peloso, A. Riotto and I. Tkachev, hep-ph/9905242; T. Asaka, K. Hamaguchi, M. Kawasaki and T. Yanagida, hep-ph/9906366.

[11] B.A. Campbell, S. Davidson and K.A. Olive, Nucl. Phys. B399 (1993) 111; H. Murayama and T. Yanagida, Phys. Lett. B322 (1994) 349 ; H. Murayama, H. Suzuki, T. Yanagida and J. Yokoyama, Phys. Rev. Lett. 70 (1993) 1912, Phys. Rev. D50 (1994) 2356 .

[12] M.Y. Khlopov and A.D. Linde, Phys. Lett. B138 (1984) 265; J. Ellis, J.E. Kim and 
D.V. Nanopoulos, Phys. Lett. B145 (1984) 181; M. Kawasaki and T. Moroi, Prog. Theor. Phys. 93 (1995) 879; see also, for example, a recent analysis, E. Holtmann, M. Kawasaki, K. Kohri and T. Moroi, Phys. Rev. D60 (1999) 023506.

[13] M. Bolz, W. Buchmüller and M. Plümacher, Phys. Lett. B443 (1998) 209.

[14] H. Pagels and J.R. Primack, Phys. Rev. Lett. 48 (1982) 223.

[15] T. Asaka, K. Hamaguchi, M. Kawasaki and T. Yanagida, in Ref. [10].

[16] C.D. Froggatt and H.B. Nielsen, Nucl. Phys. B147 (1979) 277.

[17] J. Sato and T. Yanagida, Talk given at 18th International Conference on Neutrino Physics and Astrophysics (NEUTRINO 98), (Takayama, Japan, 1998), hep-ph/9809307; P. Ramond, Talk given at 18th International Conference on Neutrino Physics and Astrophysics (NEUTRINO 98), (Takayama, Japan, 1998), hep-ph/9809401.

[18] W. Buchmüller and T. Yanagida, Phys. Lett. B445 (1999) 399.

[19] L. Covi, E. Roulet and F. Vissani, Phys. Lett. B384 (1996) 169; M. Flanz, E.A. Paschos and U. Sarkar, Phys. Lett. B345 (1995) 248; Phys. Lett. B384 (1996) 487 (E).

[20] W. Buchmüller and M. Plümacher, Phys. Lett. B431 (1998) 354.

[21] S.Y. Khlebnikov and M.E. Shaposhnikov, Nucl. Phys. B308 (1988) 885; J.A. Harvey and M.S. Turner, Phys. Rev. D42 (1990) 3344.

[22] G. Lazarides, in Ref. [10].

[23] G. Dvali, Q. Shafi and R.K. Shaefer, Phys. Rev. Lett. 73 (1994) 1886.

[24] E.J. Copeland, A.R. Liddle, D.H. Lyth, E.D. Stewart and D. Wands, Phys. Rev. D49 (1994) 6410.

[25] For a review, E.W. Kolb and M.S. Turner, The Early Universe (Addison-Wesley, 1990).

[26] C. Panagiotakopoulos, Phys. Rev. D55 (1997) 7335; A. Linde and A. Riotto, Phys. 
Rev. D56 (1997) 1841.

[27] C.L. Bennett et al., Astrophys. J. 464 (1996) L1.

[28] A.D. Linde, Phys. Lett. 108B (1982) 389; A. Albrecht and P.J. Steinhardt, Phys. Rev. Lett. 48 (1982) 1220.

[29] K.I. Izawa and T. Yanagida, Phys. Lett. B393 (1997) 331.

[30] A.D. Linde, Particle Physics and Inflationary Cosmology, (Harwood, Chur, Switzerland, 1990).

[31] K.I. Izawa, M. Kawasaki and T. Yanagida, Phys. Lett. B411 (1997) 249.

[32] T. Asaka, M. Kawasaki and M. Yamaguchi, hep-ph/9906365.

[33] A. Linde, Phys. Lett. B327 (1994) 208; A. Vilenkin, Phys. Rev. Lett. 72 (1994) 3137.

[34] K.I. Izawa, M. Kawasaki and T. Yanagida, Prog. Theor. Phys. 101 (1999) 1129.

[35] N. Sakai, H. Shinkai, T. Tachizawa and K. Maeda, Phys. Rev. D53 (1996) 655;(E) Phys. Rev. D54 (1996) 2981; I. Cho and A. Vilenkin, Phys. Rev. D56 (1998) 7621; A.A. de Laix, M. Trodden and T. Vachaspati Phys. Rev. D57 (1998) 7186.

[36] http://map.gsfc.nasa.gov/; http://astro.estec.esa.nl/SA-general/Projects/Planck.

[37] For a review, e.g., G.F. Giudice and R. Rattazzi, hep-ph/9801271.

[38] T. Moroi, H. Murayama and M. Yamaguchi, Phys. Lett. B303 (1993) 289.

[39] B. Allen, R.R. Caldwell, S. Dodelson, L. Knox, E.P.S. Shellard, and A. Stebbins, Phys. Rev. Lett. 79 (1997) 2624.

[40] T. Asaka, K. Hamaguchi, M. Kawasaki and T. Yanagida, in preparation. 